\title{
Pullout tests on the connection to an existing foundation of a steel warehouse rebuilt after the 2012 Emilia (Italy) earthquakes
}

\author{
Fabio Minghini ${ }^{1}$ (D) $\cdot$ Francesco Lippi ${ }^{2} \cdot$ Nerio Tullini $^{1} \cdot$ Walter Salvatore ${ }^{2}$
}

Received: 19 December 2020 / Accepted: 13 May 2021 / Published online: 27 May 2021

(c) The Author(s) 2021

\begin{abstract}
The tests described in this paper were aimed at evaluating the tensile capacity of the anchorages connecting an automated pallet warehouse with an existing RC foundation. The warehouse is a new steel structure erected in the place of a previous warehouse collapsed due to the Emilia earthquake, but whose foundation remained undamaged. The investigated fastening consists of 10 post-installed, bonded threaded rods with diameter $(d)$ and embedment depth $\left(h_{\mathrm{ef}}\right)$ of 20 and $500 \mathrm{~mm}$, respectively. Neither anchor arrangement nor embedment depth $\left(h_{\mathrm{ef}}>20 d\right)$ was covered by current standards for fastening design. To reproduce the in-situ actual conditions of the fastening, an unconfined test configuration was used. The maximum loads achieved were more than 3 times greater than the seismic demand for the fastening. The tests highlighted the crucial role played by the reinforcing steel which was present in the foundation. Concrete-related failure mechanisms, such as the combined pullout and concrete cone failure mechanism typical of bonded anchors, were not activated. The observed crack patterns rather suggest the onset of a flexural failure mechanism of the concrete slab. This feature is confirmed by analytical calculations showing that, at the maximum loads achieved in the tests, the top reinforcement was likely to be yielded. In six preliminary unconfined tension tests on single anchors, steel rod failure was achieved, associated with limited cracking of the concrete surface in proximity of the anchor.
\end{abstract}

Keywords Bonded anchor · Post-installed anchor - Anchor group - Cyclic tension test · Role of reinforcement $\cdot$ Failure mode

Fabio Minghini

fabio.minghini@unife.it

1 Engineering Department, University of Ferrara, Via G. Saragat 1, 44122 Ferrara, Italy

2 Department of Civil and Industrial Engineering, University of Pisa, 56122 Largo L. Lazzarino, Italy 


\section{Introduction}

\subsection{Problem statement}

In Italy, a strong impulse to use heavy-load anchors in concrete came immediately after the 2012 Emilia seismic sequence. Those earthquake events draw attention to the serious deficiencies of precast Reinforced Concrete (RC) buildings not conceived to resist seismic loads (Bournas et al. 2014; Braga et al. 2014; Minghini et al. 2016; Buratti et al. 2017). In these structures, numerous of the observed collapses due to the unseating of main girders or roof elements from their supports must be ascribed to the inadequacy of frictionbased connections (Liberatore et al. 2013; Magliulo et al. 2014). Therefore, following the Emilia earthquakes many researchers were involved in developing ductile connection systems suited for new earthquake-resistant precast structures (Parastesh et al. 2014; Tullini and Minghini 2016, 2020) or innovative dissipating devices to retrofit existing buildings (Belleri et al. 2017; Magliulo et al. 2017; Pollini et al. 2018). However, during that seismic sequence the emergency situation imposed to rapidly retrofit all buildings with inadequate connections to avoid further collapses. Structural interventions were then massively aimed at preventing relative displacements between the various monolithic elements using more traditional and cheaper techniques. These techniques were generally based on steel plates or profiles fastened to concrete by mechanical or adhesively bonded anchors. This led to an extensive use of post-installed anchors throughout a large part of Northern Italy (Minghini and Tullini 2021) and raised the issue of investigating their response in precast RC structures subjected to cyclic loads (Dal Lago et al. 2017).

\subsection{Literature review}

The possible failure modes of single chemical anchors post-installed in concrete are well documented in the literature (Cook 1993; Cook et al. 2007; Eligehausen et al. 2006a, b). In particular, for a bonded anchor subjected to tensile load under unconfined conditions and in the absence of edge effects, the unrestricted formation of a concrete cone rupture would in theory be allowed. However, due to the stress transfer between anchor and concrete along the entire bonded length, the most frequently observed failure mode consists in the formation of a concrete cone of limited depth associated with the rupture of the anchor-concrete interface bond. This failure mode is usually referred to as combined pullout and concrete failure (FIB 2011; Mallée et al. 2013). The bond rupture may be located at the interface between resin and bore wall, or between resin and threaded rod, or, finally, be a combination of the previous two.

The above quoted references form a fundamental technical background for anchor design. That said, it is known that anchors to be used in seismic areas requires a specific qualification. In Europe, numerous researches have recently been oriented to the seismic qualification of post-installed anchors (Nuti and Santini 2008; Hoehler and Eligehausen 2008a, b; Franchi et al. 2009; Hoehler et al. 2011; Guillet 2011; Wood and Hutchinson 2013; Hutchinson and Wood 2013; Mahrenholtz et al. 2016, 2017a). From these studies, the following main conclusions can be drawn. First, the loading rate typical of earthquakes does not influence the anchor capacity and qualification tests may be based on quasi-static protocols (Hoehler et al. 2011). Moreover, the anchor capacity obtained from cyclic tension tests in cracked concrete resulted comparable with that obtained from corresponding 
monotonic tests. Nevertheless, stepwise-increasing cyclic load protocols were found the best choice for safely reproducing in the experiments the seismic response of anchors (Hutchinson and Wood 2013; Mahrenholtz et al. 2016). The presence of a crack in concrete in correspondence of an anchor strongly reduces the anchor capacity. In particular, the cyclic variation of crack opening occurring during an earthquake leads to a rapid strength degradation of the anchorage (Nuti and Santini 2008; Hoehler and Eligehausen 2008a; Franchi et al. 2009). Therefore, the use of a stepwise-increasing cyclic crack protocol was proposed (Mahrenholtz et al. 2017a). That work showed that bonded anchors are particularly affected by cycling crack opening. Then, these anchors may show an acceptable response if their seismic design strength is properly reduced with respect to the non-seismic design strength. Finally, for anchors subjected to combined tensile and shear seismic forces, a linear interaction diagram was recommended in the absence of specific experimental tests (Guillet 2011). These findings flowed into the seismic qualification procedure and design method reported by EOTA (European Organisation for Technical Assessment) 2013a, b; 2016b). The technical basis for the seismic qualification of anchors were recently summarized by Mahrenholtz et al. (2017b), which also proposed a unified testing protocol for the assessment of anchors at multiple performance levels.

Compared with the investigations on single anchors, fewer studies concerning the behaviour of anchor groups in tension are available. In particular, some recent numerical investigation was aimed at estimating capacity (Ozturk 2013) and failure pattern (Ballarini and Yueyue 2017) of anchor groups under tensile loads. With regard to experimental investigations, 28 tests on anchor groups loaded monotonically by various combinations of moment and shear were presented by Cook and Klingner (1992). In those tests the failure was controlled by the strength of the anchor steel and a behavioural model based on the limit design theory was then proposed by the authors. The cyclic tension behaviour of groups of cast-in-place anchors was addressed by Delhomme et al. (2015). The specimens analyzed in that research included a steel plate connected with a RC foundation slab using four deformed steel bars or four headed anchor rods, positioned within the reinforcement cage of the foundation prior to casting the concrete. In monotonic tension tests without edge effects, the steel failure of the anchors was always attained. In cyclic tension tests on bonded bars, due to the large embedment depth $\left(h_{\mathrm{ef}}=24 d\right.$, with $d$ being the anchor diameter), a progressive bond failure associated with bar yielding was observed. Moreover, both cyclic and monotonic tensile capacities were not particularly affected by concrete cracking. A theoretical study on groups of four cast-in-place headed anchors embedded in narrow RC members was presented by Węglorz (2017). The concrete breakout failure of groups of 6 embedded anchors was investigated by Grilli and Kanvinde (2015). There is no element to justify extending the findings reported by Delhomme et al. (2015), Grilli and Kanvinde (2015) and Węglorz (2017) to the case of groups of post-installed, adhesively bonded anchors. The experimental investigation described by Liu et al. (2014) is only partly concerned with groups of four bonded anchors in tension. Arslan (2016) tested in tension groups of three anchors comprised of bonded rebars or threaded rods, and highlighted the influence of the edge distances on the failure mode. The influence of anchor diameter, spacing and embedment depth on the behaviour of groups of 4 adhesive anchors in tension was investigated by Lehr (2003). Monotonic tension and shear tests on groups of 4, 6 and 9 adhesive anchors were presented by Epackachi et al. (2015), which confirmed the validity of the design equations proposed by Eligehausen et al. (2006a). A very interesting experimental study concerning concrete cone failure of anchor groups with geometric configurations not covered by current design provisions was presented by Bokor et al. (2019a). The fastenings were loaded in monotonic tension. The influence of load eccentricity and 
stiffness of the base plate was also investigated. An efficient spring model was then proposed by the same authors (Bokor et al. 2019b) to interpret the experimental results.

The consolidated design method for anchors in tension (EOTA 1997, 2007; FIB 2011; Mallée et al. 2013) covers groups of up to nine anchors. In particular, TR 29 by EOTA (2007), tailored to bonded anchors, modifies Annex C of ETAG 001 (EOTA 1997) to include the combined pullout and concrete failure. These documents take account of the adverse influence of a dense surface reinforcement (shell spalling) when the bonded anchors are anchored in the concrete cover or near the reinforcement. The only design recommendations on the amount of concrete reinforcement are concerned with headed anchors. This reinforcement should include bars parallel to the anchors, designed to resist the whole tensile load, as well as surface bars, designed to resist the splitting forces (see Fig. 4.17b reported by Mallée et al. 2013). With regard to this topic, a recent experimental research (Nilforoush et al. 2018) highlighted the influence of surface reinforcement in the case of headed anchors in uncracked concrete failing by concrete breakout. In particular, it was shown that, due to surface reinforcement, the fastening behaviour becomes more ductile and the failure load increases on average of $17 \%$ and $6 \%$ for a thickness of the concrete member equal to $1.5 h_{\mathrm{ef}}$ and $3.0 h_{\mathrm{ef}}$, respectively. Similar conclusions were also drawn based on a numerical study (Nilforoush et al. 2017).

Very recently, EN 1992-4 (CEN 2018) collected the above mentioned design guidelines into one single document, becoming one of the most up-to-date standards on fastenings' design available all over the world. Anchorage configurations of up to nine anchors are covered also in this case. Moreover, in analogy with the recommendations reported by EOTA (2007), FIB (2011) and Mallée et al. (2013), the embedment depth for bonded anchors must satisfy the condition $h_{\mathrm{ef}} \leq 20 \mathrm{~d}$. In fact, the assumption of uniform bond stresses along $h_{\text {ef }}$, usually adopted to estimate the pullout strength, cannot be considered valid for arbitrarily large embedment depths.

In the case of post-installed steel rebars, many researches showed that two different design philosophies can alternatively be followed (Mahrenholtz 2012; Mahrenholtz et al. 2014, 2015; Mahrenholtz and Eligehausen 2016): (1) the design method for bonded anchors or (2) the development length theory for reinforcing bars (EOTA 2018). For the bonded rebars investigated by Mahrenholtz et al. (2015), a value of the embedment depth beyond which the failure load remains constant was found by the authors. This embedment depth, corresponding to the achievement of bar yielding, is smaller for single bars than for groups of bars, for which the failure is dominated by concrete breakout in a wide range of $h_{\text {ef }}$ values. To the writers' knowledge, there is a lack of analogous research findings focusing on post-installed, bonded threaded rods.

\subsection{Scope of the work}

In this paper, an experimental investigation on groups of ten adhesively bonded threaded rods, post-installed into RC foundation slabs, is presented. The experiments represent a case study intended to reproduce the cyclic tension behaviour of the anchorages connecting an automated pallet storage warehouse with an existing RC foundation. The warehouse is a new steel structure, erected in the place of a previous warehouse collapsed during the first mainshock of the Emilia earthquakes (see Figs. 1, 2). In the seismic design of the new warehouse, a unitary behaviour factor was adopted. Therefore, even during the design earthquake with return period of 475 years, the structure and its anchorage to the foundation are presumed to display an elastic behaviour. In fact, the capacity design requirements 


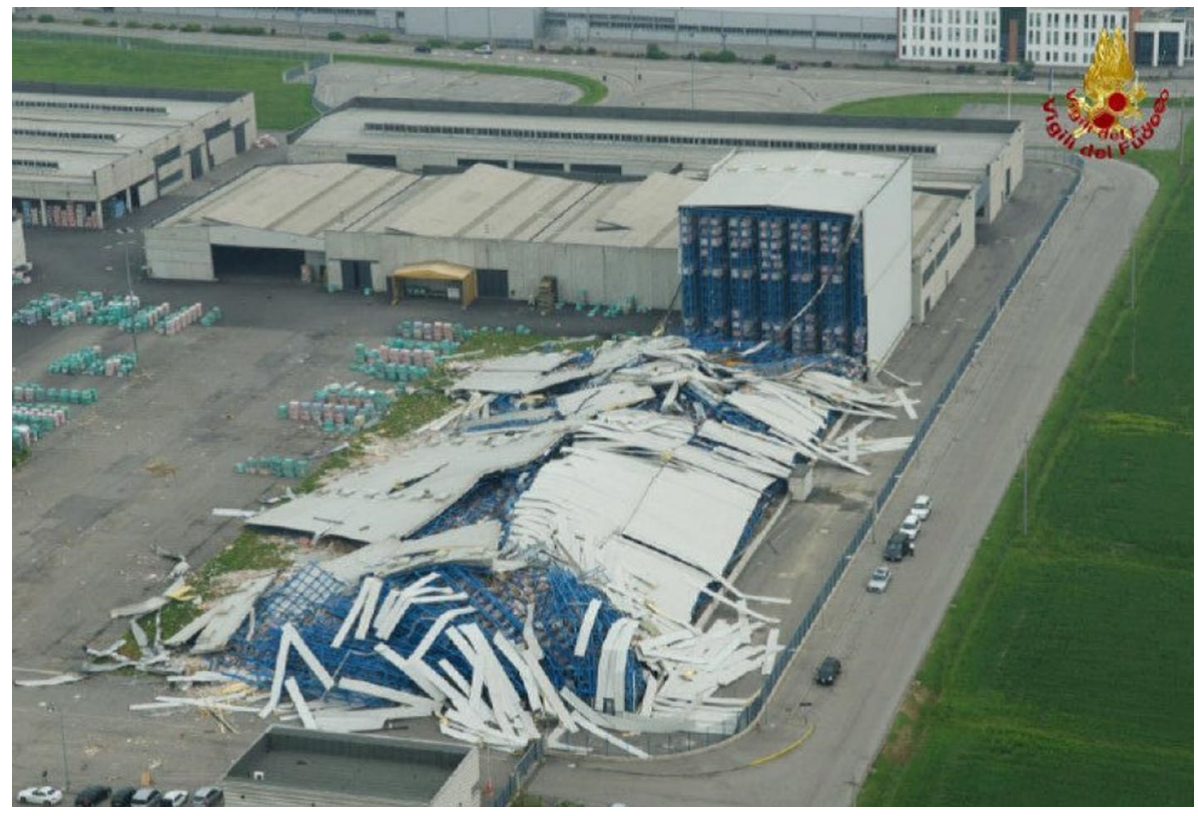

Fig. 1 Aerial view of the struck industrial area showing the collapsed warehouse (courtesy of the Italian National Fire Corps)

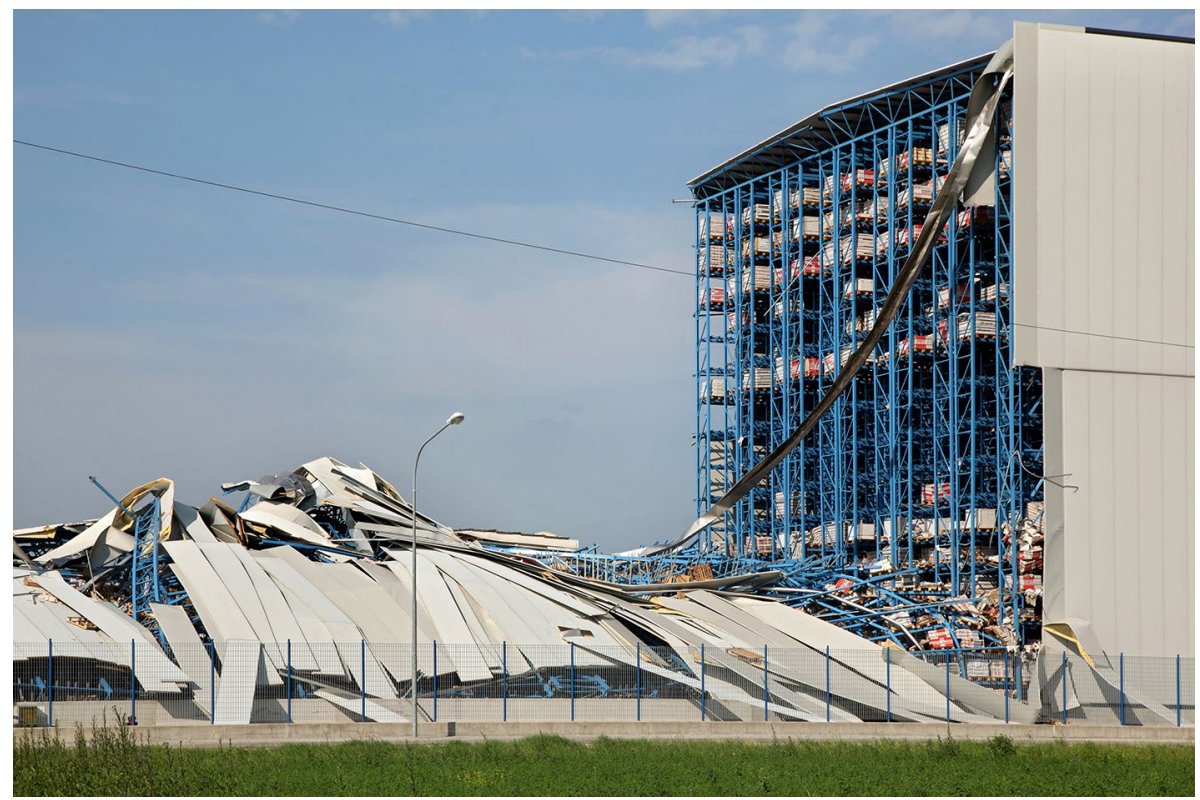

Fig. 2 Close-up view of the collapsed racks (picture by Paolo Righi; copyright: Meridiana Immagini) 
are not always applicable to this kind of structures (Caprili et al. 2018). Being the existing foundation undamaged, uncracked concrete conditions were considered at the beginning of tests.

The main objective of this research was to be supportive for the design of the anchorage of the pallet warehouse to the foundation. In fact, rods arrangement and embedment depth prescribed for the groups of bonded anchors were not covered by consolidated design methods (EOTA 2007; FIB 2011; Mallée et al. 2013; CEN 2018). Therefore, an experimental characterization of the anchorage response was necessary to validate the design. Two unconfined tension tests on anchor groups were carried out with stepwise-increasing cyclic load protocol. At the end of tests, in which extensive concrete cracking was achieved, a monotonic tension test was carried out on one of the specimens to check its residual capacity. The experimental load-displacement plots and failure modes are reported and discussed. Finally, an interpretation of the experimental findings, highlighting the contribution of the foundation reinforcement, is proposed. These experiments were preceded by eight unconfined tension tests on single anchors. Four of these tests were carried out under monotonic tension, whereas in the remaining four tests a cyclic tensile force was applied.

\section{Fastening of the new structure to the existing foundation}

The new steel warehouse rises in the place of a structure largely destroyed by the first mainshock of the Emilia earthquake sequence, occurred in Northern Italy on May $20^{\text {th }}$, 2012, with $\mathrm{M}_{\mathrm{L}}=5.9$ (Figs. 1,2). That collapse, largely documented in the literature (Carydis et al. 2012; Liberatore et al. 2013; Bournas et al. 2014; Kanyilmaz et al. 2016a, b; Formisano et al. 2017), highlighted the critical issues related with unbraced steel storage racks under seismic action. In fact, the old structure was designed to support heavy dead loads resulting from the storage of ceramic products, but was not provided with bracing systems adequate to resist earthquake loads. In some cases, such as in this one, failure is the consequence of column uplifting on one side associated with significant column buckling on the other side. Uplifting was caused by a largely insufficient tensile capacity of the column base steel plate-to-foundation connections compared with earthquake-induced vertical tensile forces. In other cases, failure may be caused by a soft storey mechanism due to an insufficient stiffness of base plate connections (Kanyilmaz et al. 2016b). With regard to these topics, two relevant EU-RFCS Projects were carried out (see reports by Rosin et al. 2009; Castiglioni et al. 2014), which investigated the behaviour of base plate connections of racking systems, including push-over and shaking table tests, and led to analogous results. The interested reader is also referred to the recent contributions by Castiglioni et al. (2015) and Castiglioni (2016). Recently, shaking table tests were carried out (Maguire et al. 2020) to compare three different types of column-to-foundation connections: anchorages using (1) ductile or (2) stiffer base plates, and (3) completely unanchored base plates. Whilst in the last case rocking is likely to occur, involving reductions in the stress state at the column base but also the risk of overturning, the use of heavy duty base plates may lead to anchors pullout and significant foundation concrete damage. Conversely, ductile base plates preserve foundation from damage and seem then advantageous.

In any case, it appears evident that concrete-related failure mechanisms of the foundation should be avoided to ensure an efficient response of storage racks to strong earthquakes.

The existing $\mathrm{RC}$ foundation is comprised of a $700 \mathrm{~mm}$-thick rectangular slab on $1 \mathrm{~m}$-diameter piles spaced by $4 \mathrm{~m}$ into two orthogonal directions. During the earthquake, 
the steel structure failed prematurely, but the foundation resulted undamaged. Therefore, after removal of the collapsed structure, the new warehouse was erected on the existing foundation.

The new warehouse (Fig. 3) was erected in only 12 months. For the structure and its cladding, $2400 \mathrm{t}$ of steel profiles and $8700 \mathrm{~m}^{2}$ of sandwich panels were used, respectively. All profiles are made of steel S355JR, with nominal yield and ultimate strengths $f_{\mathrm{y}}=355 \mathrm{MPa}$ and $f_{\mathrm{t}}=510 \mathrm{MPa}$. The warehouse has in-plan dimensions of $134 \mathrm{~m} \times 26 \mathrm{~m}$, and a total height of about $18 \mathrm{~m}$. Its structural skeleton is comprised of seven $16 \mathrm{~m}$-high racks, each of them having 13 loading levels. The two peripheral racks have a width of $1155 \mathrm{~mm}$ and present one single pallet place per each level. The five inner racks have a width of $2310 \mathrm{~mm}$ and present two pallet places per each level. The total storage capacity is of 18,852 pallet places. From the structural point of view, the warehouse was conceived as a truss structure, with centroidal axes of profiles converging into one single point at each node and profiles mainly subjected to axial load. The resistance to lateral loads is ensured by concentric bracing systems.

Each column of the warehouse is anchored to the foundation by means of a group of adhesively bonded $20 \mathrm{~mm}$-diameter threaded rods with embedment depth of $500(=25 d)$ or $550 \mathrm{~mm}(=27.5 d)$. These anchorages, as well as the structure, were designed against seismic actions assuming a behaviour factor $q=1$, so that they should respond to the Ultimate Limit State (ULS) design earthquake with no significant damage.

The most critical situation for the base anchorages occurs when the horizontal component of the earthquake load is considered acting in the racks transverse direction. In particular, the most stressed anchorages are those corresponding to the lateral columns of the five inner racks, which are indicated by red arrows in Fig. 3. One of these anchorages, including ten bonded anchors, is highlighted in Fig. 4. In the presence of wind or

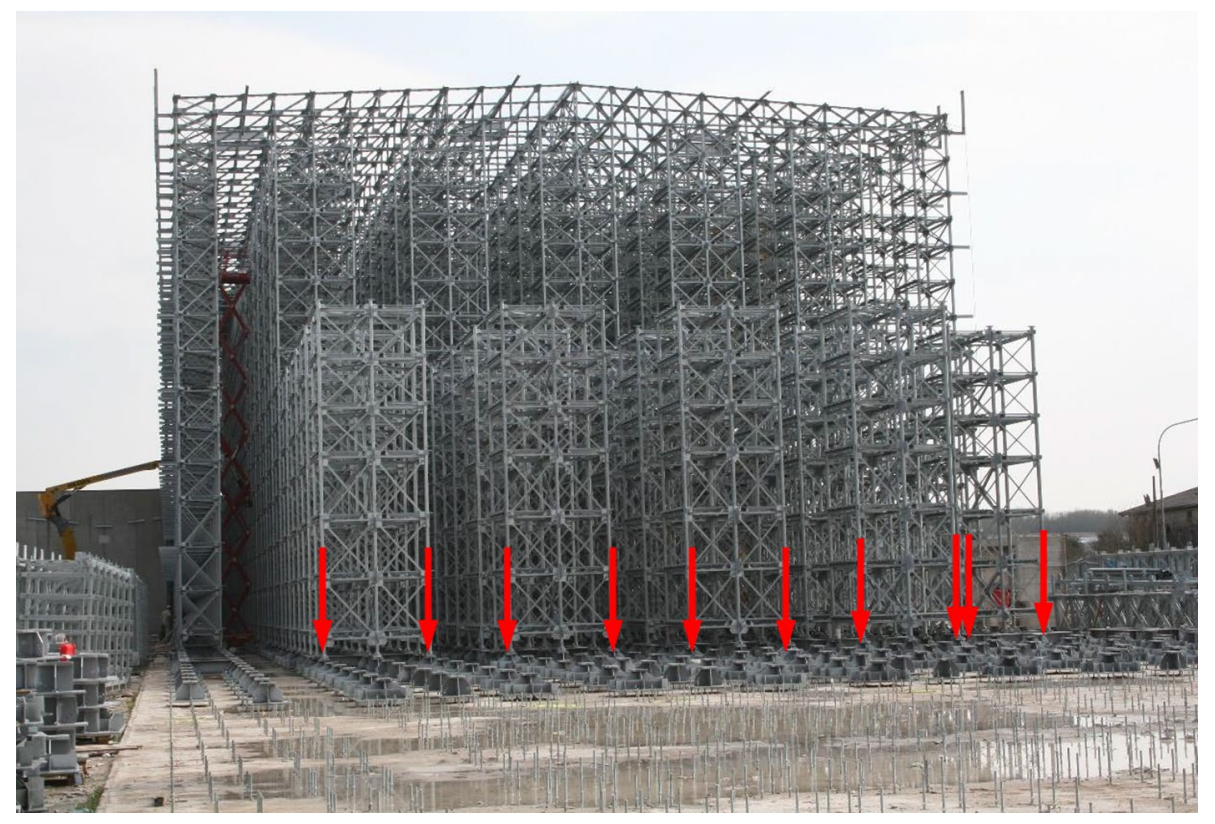

Fig. 3 The new warehouse under construction. The anchor groups tested are identical to the base anchorages for all lateral columns of the five inner racks (indicated by red arrows) 


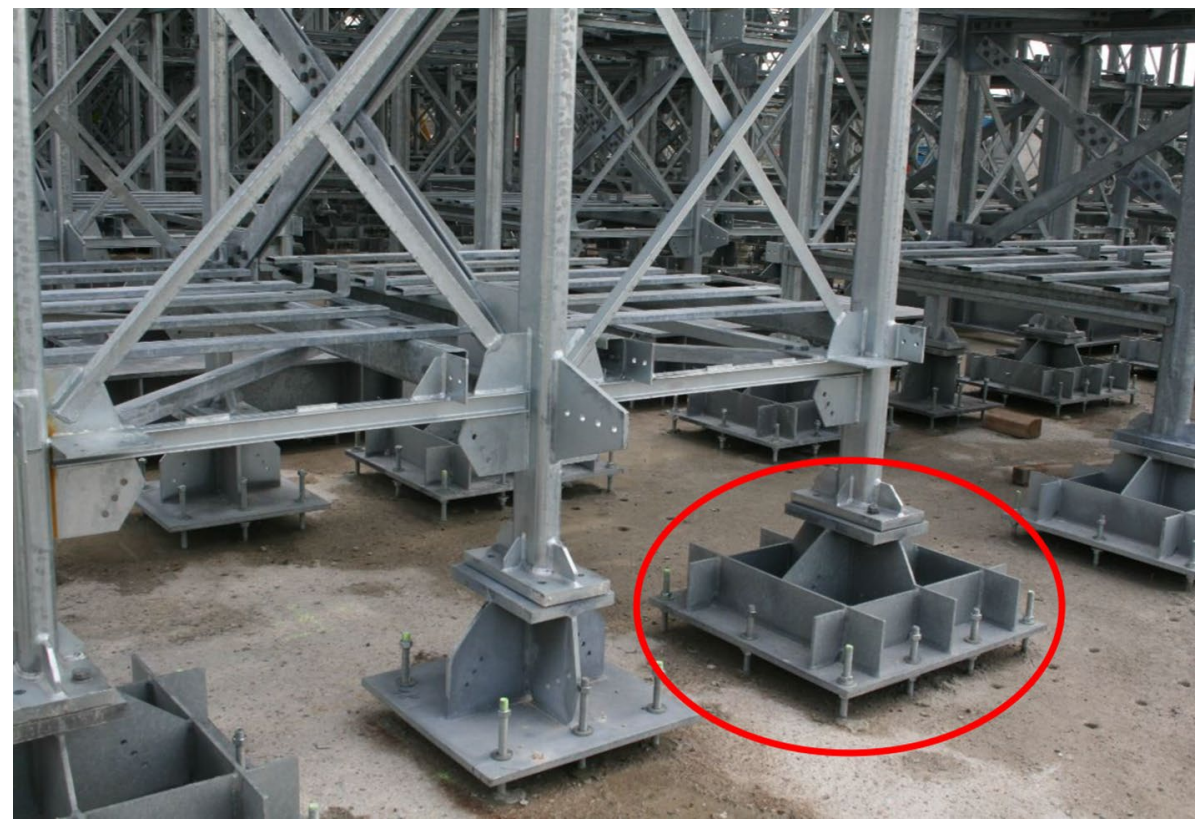

Fig. 4 Detail of a base anchorage with a group of ten bonded anchors

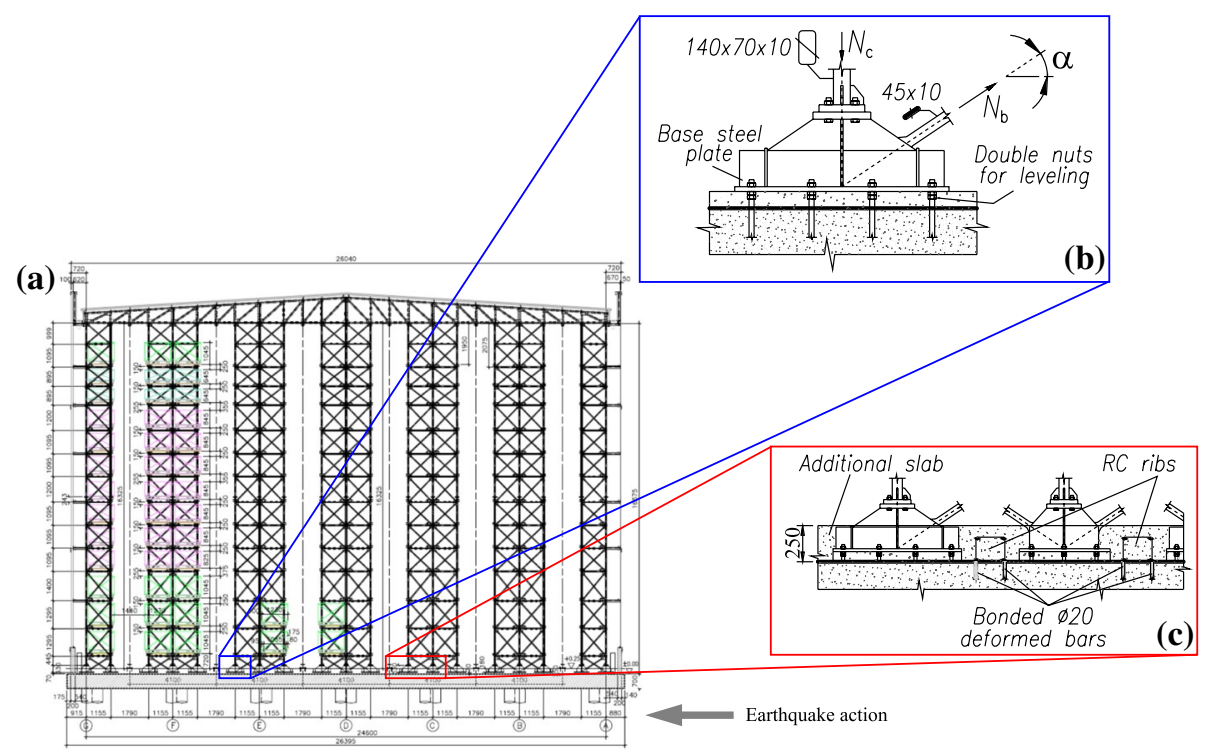

Fig. 5 Earthquake effects on the base anchorages: a transverse sectional view of the new warehouse; b schematic for the evaluation of actions on the anchor groups for lateral columns of inner racks; $\mathbf{c}$ RC ribs, placed between fastenings, used to resist shear forces 
earthquake loads acting in the racks transverse direction (Fig. 5a), the anchorage should resist the tensile force (Fig. 5b):

$$
N_{\text {Ed }}=N_{\mathrm{b}} \sin \alpha-N_{\mathrm{c}},
$$

with $N_{\mathrm{c}}$ and $N_{\mathrm{b}}$ being the axial loads in column and tension bracing, respectively. To resist shear force $V_{\mathrm{Ed}}$, RC ribs were cast on the existing foundation and anchored to it (Fig. 5c) between the base plates of the steel structure. Therefore, the anchor groups should be designed against tensile force $N_{\mathrm{Ed}}$ only. The maximum tensile force to be resisted by the group of ten bonded anchors is obtained under earthquake loading and equals $N_{\mathrm{Ed}}=539$ $\mathrm{kN}$.

\subsection{Outline of the experimental activity}

The connection of the new warehouse to the existing foundation was obtained from $20 \mathrm{~mm}$ diameter threaded rods inserted into $24 \mathrm{~mm}$-diameter drilled holes, previously injected with epoxy resin. To characterize these anchorages, eleven in-situ tests were carried out (Table 1).

For tests on the group of ten anchors (No. 9 to 11 in Table 1), identified with G1 to G3, an embedment depth $h_{\mathrm{ef}}=500 \mathrm{~mm}$ was used. These tests were preceded by single-anchor tests (No. 1 to 8 in Table 1), identified with $\mathrm{S} n-h_{\mathrm{ef}}-X$, where $n$ indicates the test number, $h_{\mathrm{ef}}$ is the embedment depth, equal to either 500 or $550 \mathrm{~mm}$, and $X=\mathrm{M}$ or $\mathrm{C}$ for monotonic or cyclic loading, respectively.

Table 1 Matrix of experiments and results

\begin{tabular}{|c|c|c|c|c|c|c|c|}
\hline Test \# & Test ID ${ }^{\mathrm{a}}$ & $\begin{array}{l}d / d_{0} / d_{\mathrm{fix}}^{\mathrm{b}} \\
(\mathrm{mm}) /(\mathrm{mm}) /(\mathrm{mm})\end{array}$ & $\begin{array}{l}h_{\mathrm{ef}} \\
(\mathrm{mm})\end{array}$ & Loading type & Failure mode ${ }^{\mathrm{d}}$ & $\begin{array}{l}\text { Peak load } \\
(\mathrm{kN})\end{array}$ & $\begin{array}{l}\text { Maximum } \\
\text { displacement }^{\mathrm{e}} \\
(\mathrm{mm})\end{array}$ \\
\hline 1 & S1-550-M & 20/24/- & 550 & Monotonic & - & 216.7 & 3.21 \\
\hline 2 & $\mathrm{~S} 2-550-\mathrm{M}$ & 20/24/- & 550 & Monotonic & $\mathrm{S}$ & 214.2 & 4.02 \\
\hline 3 & S3-550-M & 20/24/- & 550 & Monotonic & - & 216.6 & 3.17 \\
\hline 4 & $\mathrm{~S} 4-550-\mathrm{C}$ & 20/24/- & 550 & Cyclic & $\mathrm{S}$ & 210.2 & 2.42 \\
\hline 5 & S5-500-C & 20/24/- & 500 & Cyclic & $\mathrm{S}$ & 209.2 & 2.80 \\
\hline 6 & S6-500-M & 20/24/- & 500 & Monotonic & $\mathrm{S}$ & 216.6 & 5.70 \\
\hline 7 & S7-500-C & 20/24/- & 500 & Cyclic & $\mathrm{S}$ & 212.6 & $1.59 / 0.52$ \\
\hline 8 & S8-500-C & 20/24/- & 500 & Cyclic & $\mathrm{S}$ & 213.4 & $5.83 / 2.75$ \\
\hline 9 & G1 & $20 / 24 / 22$ & 500 & Cyclic & $\mathrm{Y}$ & 1627.1 & $2.84 / 2.56$ \\
\hline 10 & $\mathrm{G} 2^{\mathrm{c}}$ & $20 / 24 / 22$ & 500 & One cycle & $\mathrm{Y}$ & 1655.6 & $4.44 / 3.37$ \\
\hline 11 & G3 & $20 / 24 / 22$ & 500 & Cyclic & $\mathrm{Y}$ & 1735.6 & $5.20 / 3.01$ \\
\hline
\end{tabular}

${ }^{\mathrm{a}}$ For all tests, the thickness of the concrete foundation was $h=700 \mathrm{~mm}$

${ }^{\mathrm{b}}$ Diameters of threaded rod $(d)$, drill bit $\left(d_{0}\right)$ and clearance hole in the fixture $\left(d_{\text {fix }}\right)$

${ }^{\mathrm{c}}$ Specimen previously subjected to test G1. One single loading cycle performed

${ }^{\mathrm{d}} \mathrm{S}$ and $\mathrm{Y}$ stand for Steel rod failure and reinforcement Yielding, respectively. Occurrence of failure mode $\mathrm{Y}$ was supposed based on calculations reported in Table 7

' Tests \#1 to \#6: $\left(u_{\mathrm{dg} 1}+u_{\mathrm{dg} 2}\right) / 2$. Tests \#7 and \#8: $u_{\mathrm{dg} 1}(\operatorname{rod}) / u_{\mathrm{dg} 2}$ (concrete); Tests \#9 to \#11: $\left[\left(u_{\mathrm{dg} 1}+u_{\mathrm{dg} 2}+u_{\mathrm{dg} 3}+u_{\mathrm{dg} 4}\right) / 4\right] /\left[\left(u_{\mathrm{dg} 5}+u_{\mathrm{dg} 6}\right) / 2\right]$ 
For all of the tests, an unconfined configuration was adopted. In order to limit the testinduced damage to the foundation, it was decided to bond to the existing RC slab only the threaded rods used for single-anchor tests. The locations of these tests were established at about $2 \mathrm{~m}$ from one of the slab edges and $2.4 \mathrm{~m}$ from the closest columns of the new steel racks. For tests on anchor groups, two new $700 \mathrm{~mm}$-thick concrete basements were realized. They were square in plan with the side of $3.8 \mathrm{~m}$ and presented a reinforcement ratio equal to the minimum reinforcement ratio of the existing slab.

\subsection{Material properties}

The main properties of materials involved in the present research are reported in Table 2.

The bonded anchors used in the tests were produced by the same manufacturer and presented the same properties as those used for the base anchorages for the new warehouse. These properties are declared in the relevant European Technical Approval (ETA). It is worth observing that the anchors were qualified for seismic performance category $\mathrm{C} 2$, the most stringent one. However, according to the ETA, and to FIB (2011) and CEN (2018), $h_{\mathrm{ef}}$ for $20 \mathrm{~mm}$-diameter threaded rods should range between $90 \mathrm{~mm}\left(4.5 h_{\mathrm{ef}}\right)$ and $400 \mathrm{~mm}$ $\left(20 h_{\mathrm{ef}}\right)$. Therefore, the choice of using, for the anchorages shown in Fig. 4, embedment depths of 500 or $550 \mathrm{~mm}$ required the experimental characterization illustrated in the present paper. The anchors were installed according to the manufacturer's installation instructions by appropriately qualified personnel.

The tensile strength of the threaded rods used for the anchorages was estimated based on preliminary tests on 30 rod specimens. The mean tensile resistance obtained resulted $8 \%$ greater than the characteristic resistance reported in the ETA for steel failure, whereas the coefficient of variation was $2.6 \%$.

The concrete compressive strength for the two basements used for tests on anchor groups was estimated from tests on 6 cubes. According to type A acceptance check defined by IMIT (2018), minimum and mean compressive strengths reported in Table 2 are consistent with class $\mathrm{C} 25 / 30$. With regard to the existing slab, results from recent compression tests on drilled concrete cores and rebound hammer tests confirmed the results from compression tests on cubic specimens carried out at the time of construction. The concrete strength class for the slab is $\mathrm{C} 30 / 37$.

The properties of reinforcing bars used in the basements, obtained from tests on 3 steel specimens, are consistent with grade B450C (IMIT 2018).

\section{Tests setup}

This Section reports the description of test layout, loading protocols, equipment and measuring system adopted in the tests.

The experiments were aimed at evaluating the tensile capacity displayed by the fastenings in the actual installation conditions. Therefore, for all of the tests, an unconfined test setup was used according to EOTA (2016a). Moreover, the two basements of dimensions $3800 \times 3800 \times 700 \mathrm{~mm}$ used for tests on anchor groups were initially uncracked, as well as the existing slab involved by the single anchor tests. These basements were reinforced with $16 \mathrm{~mm}$-diameter deformed bars positioned on both the top and the bottom with a spacing of $250 \mathrm{~mm}$ into two orthogonal directions (Fig. 6a). The concrete cover was of $34 \mathrm{~mm}$. 
Table 2 Properties of materials used in the tests

\begin{tabular}{|c|c|c|}
\hline \multicolumn{2}{|c|}{ Symbol, description (Unit) } & \multirow[t]{2}{*}{ Value } \\
\hline Bonded anchors & Reference document: relevant ETA & \\
\hline$d$ & Threaded rod diameter $(\mathrm{mm})$ & 20 \\
\hline$d_{0}$ & Drill bit diameter $(\mathrm{mm})$ & 24 \\
\hline$d_{\text {fix }}$ & Clearance hole in the fixture $(\mathrm{mm})$ & 22 \\
\hline$h_{\mathrm{ef}}(\min / \max )$ & Embedment depth (mm) & $90 / 400$ \\
\hline$T_{\text {inst }}$ & Initial torque $(\mathrm{Nm})$ & 130 \\
\hline$\tau_{\mathrm{Rk} 20 / 25}$ & Characteristic bond strength for uncracked concrete C20/25 (MPa) & 10.0 \\
\hline \multirow[t]{5}{*}{$\tau_{\mathrm{Rk}}\left(=\tau_{\mathrm{Rk}, \mathrm{ucr}}^{\mathrm{a}}\right)$} & Characteristic bond strength, uncracked concrete (MPa) & \\
\hline & Existing foundation & 10.8 \\
\hline & Basements for tests on anchor groups & 10.4 \\
\hline & Seismic qualification, reference document: relevant ETA & \\
\hline & Seismic performance category & $\mathrm{C} 2$ \\
\hline$\tau_{\mathrm{Rk}, \mathrm{eq}}{ }^{\mathrm{a}}$ & Characteristic seismic bond strength (MPa) & 2.8 \\
\hline$N_{\text {Rk,eq }}$ & Tensile resistance, steel failure $(\mathrm{kN})$ & 196 \\
\hline$\delta_{N, \mathrm{eq}(\mathrm{DLS})}$ & Displacement for tensile load (DLS) (mm) & 0.25 \\
\hline \multirow[t]{2}{*}{$\delta_{N, \mathrm{eq}(\mathrm{ULS})}$} & Displacement for tensile load (ULS) (mm) & 0.45 \\
\hline & Estimated quantities & \\
\hline \multirow[t]{3}{*}{$\tau_{\mathrm{Rm}}\left(=\tau_{\mathrm{Rm}, \mathrm{ucr}}\right)$} & Mean bond strength, uncracked concrete $\left(=\tau_{\mathrm{Rk}}\left(f_{\mathrm{cm}} / f_{\mathrm{ck}}\right)^{2 / 3}\right)(\mathrm{MPa})$ & \\
\hline & Existing foundation & 12.6 \\
\hline & Basements for tests on anchor groups & 11.6 \\
\hline Threaded rods & Reference document: tension test report ${ }^{\mathrm{b}}$ & \\
\hline$d_{\mathrm{n}}$ & Nominal diameter $(\mathrm{mm})$ & 20 \\
\hline$N_{\mathrm{Rm}}$ & Mean tensile resistance $(\mathrm{kN})$ & 212 \\
\hline \multicolumn{3}{|c|}{ Existing foundation ${ }^{\mathrm{c}}$} \\
\hline Concrete & Reference document: compression test report & \\
\hline$f_{\text {cmin,cube }}$ & Minimum cubic compressive strength (MPa) & 34.9 \\
\hline$f_{\text {cm,cube }}$ & Mean cubic compressive strength (MPa) & 46.3 \\
\hline & Estimated quantities & \\
\hline$f_{\mathrm{cm}}$ & Mean compressive strength $\left(=0.83 f_{\mathrm{cm}, \text { cube }}\right)(\mathrm{MPa})$ & 38.4 \\
\hline$f_{\text {ck }}$ & Charact. compressive strength (from type B check, IMIT 2018) (MPa) & 30.0 \\
\hline Reinforcing steel & Reference document: tension test report & \\
\hline$\varnothing_{\mathrm{n}}$ & Nominal diameters (mm) & $14,16,22$ \\
\hline$f_{\mathrm{ym}}$ & Mean yield strength $[\mathrm{MPa}]$ & 531 \\
\hline \multicolumn{3}{|c|}{ Basements for tests on anchor groups ${ }^{\mathrm{d}}$} \\
\hline Concrete & Reference document: compression test report & \\
\hline$f_{\text {cmin,cube }}$ & Minimum cubic compressive strength (MPa) & 34.3 \\
\hline \multirow[t]{2}{*}{$f_{\text {cm,cube }}$} & Mean cubic compressive strength (MPa) & 35.4 \\
\hline & Estimated quantities & \\
\hline$f_{\mathrm{cm}}$ & Mean compressive strength $\left(=0.83 f_{\mathrm{cm}, \text { cube }}\right)(\mathrm{MPa})$ & 29.4 \\
\hline$f_{\mathrm{ck}}$ & Charact. compressive strength (from type A check, IMIT 2018) (MPa) & 25.0 \\
\hline Reinforcing steel & Reference document: tension test report & \\
\hline$\varnothing_{\mathrm{n}}$ & Nominal diameter (mm) & 16 \\
\hline$f_{\mathrm{ym}}$ & Mean yield strength (MPa) & 495 \\
\hline$f_{\mathrm{tm}}$ & Mean ultimate tensile strength (MPa) & 602 \\
\hline
\end{tabular}


Table 2 (continued)

Symbol, description (Unit)

Value

Bonded anchors Reference document: relevant ETA

\begin{tabular}{lll}
\hline$\varepsilon_{\mathrm{um}}$ & Mean ultimate tensile strain (\%) & 13.9 \\
\hline
\end{tabular}

${ }^{\mathrm{a}}$ Values relative to temperature ranging between $-40^{\circ} \mathrm{C}$ and $+40^{\circ} \mathrm{C}$ (long-term mean temperature $T_{\mathrm{mlp}}=24^{\circ} \mathrm{C}$ )

${ }^{\mathrm{b}}$ Concerning 30 specimens

${ }^{c}$ Properties deduced from the original test reports dating back at the time of construction. The reports concern 48 concrete cubic specimens with the side of $150 \mathrm{~mm}$ and 9 steel specimens, 3 for each diameter

${ }^{\mathrm{d}}$ Properties deduced from recent test reports concerning 6 concrete cubic specimens with the side of $150 \mathrm{~mm}$ and 3 steel specimens
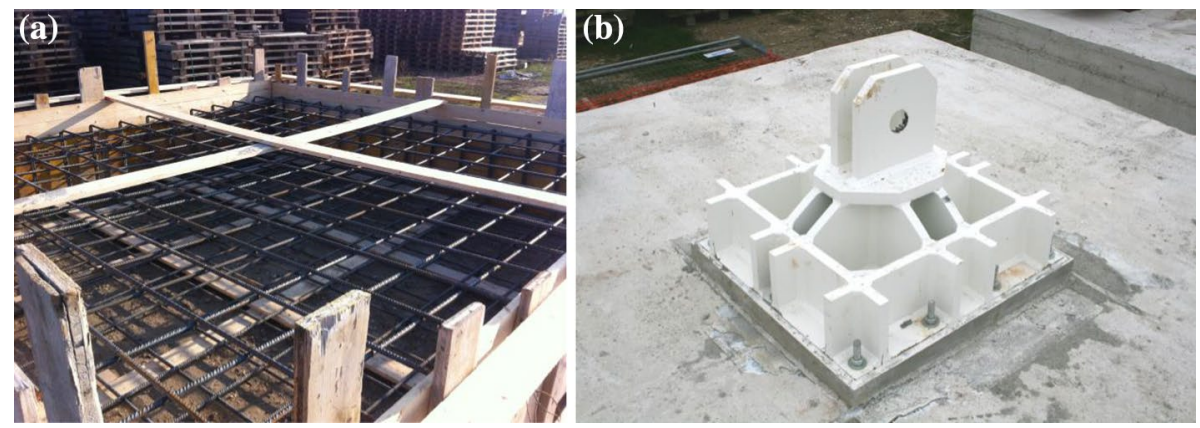

Fig. 6 Preparation for tests on anchor groups: a reinforcement cage of one of the basements and $\mathbf{b}$ steel fixture installed onto one hardened basement

In all tests the tensile load was applied quasi-statically. To avoid possible detrimental effects on the bond strength of reinforcing bars and threaded rods, strain gauges were not used in this research.

\subsection{Test layout and loading protocols}

The configurations used for tests on single anchors and anchor groups are shown in Fig. 7a, b, respectively.

With regard to tests on single anchors, unconfined test conditions were ensured by a distance of the anchor to each of the supports of the reaction frame of $1500 \mathrm{~mm}$. In fact, being the reaction frame simply supported at the ends on two beams resting on $300 \mathrm{~mm}$-wide steel plates, the anchor-to-support net distance resulted to be of $1350 \mathrm{~mm}>2 h_{\mathrm{ef}}$ for both investigated values of $h_{\mathrm{ef}}$.

A 6-cycles loading protocol was used for tests \#4 and \#5 (see Table 1). This protocol is reported in nondimensional form in Fig. 8a, where $N_{\mathrm{Rm}}$ indicates the mean tensile resistance of the threaded rods (Table 2), i.e., the expected maximum failure load. Moreover, to investigate the effects due to the number of load cycles, the 10-cycles loading protocol shown in Fig. 8b was adopted for tests \#7 and \#8.

With regard to tests on anchor groups, according to the configuration shown in Fig. 7b a constant bending moment arises in the basement in the region between the anchors closest 

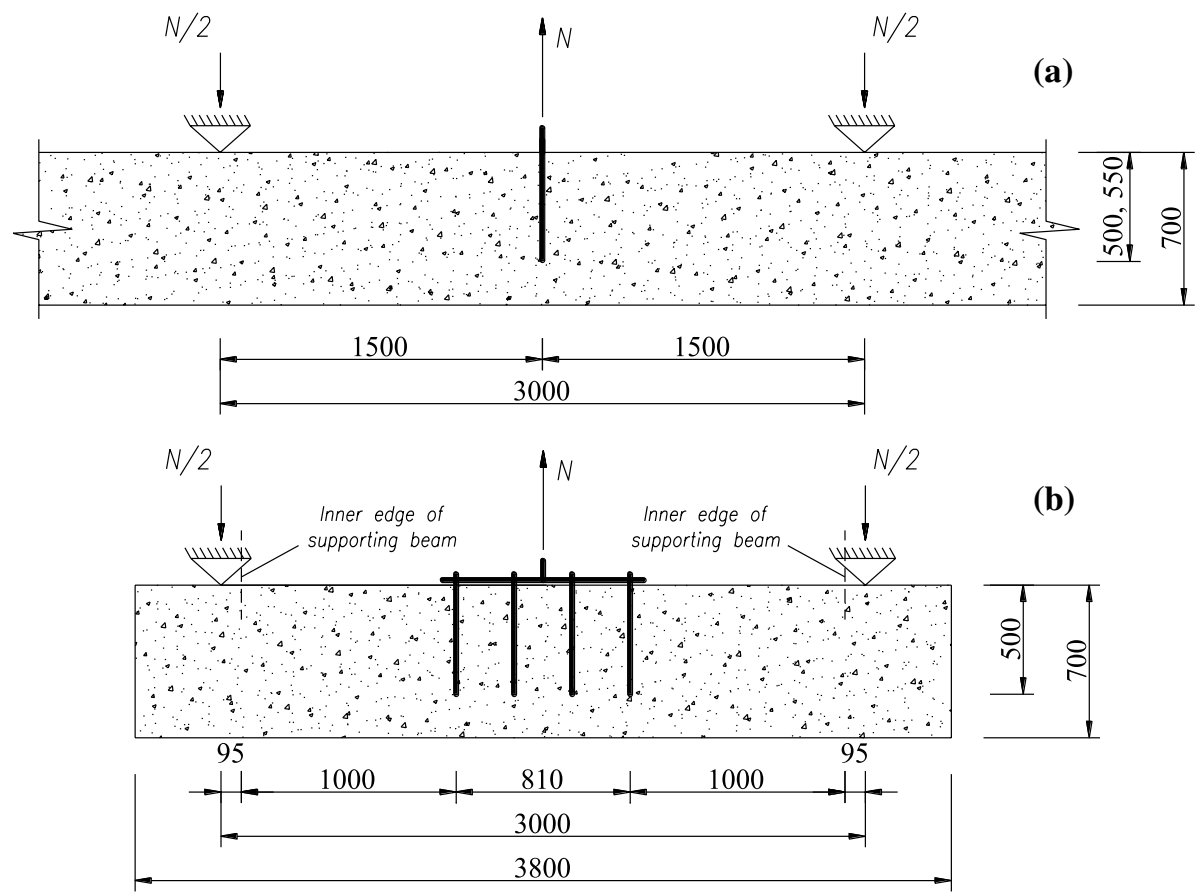

Fig. 7 Configuration for tests on $\mathbf{a}$ single anchors and $\mathbf{b}$ anchor groups

(a)

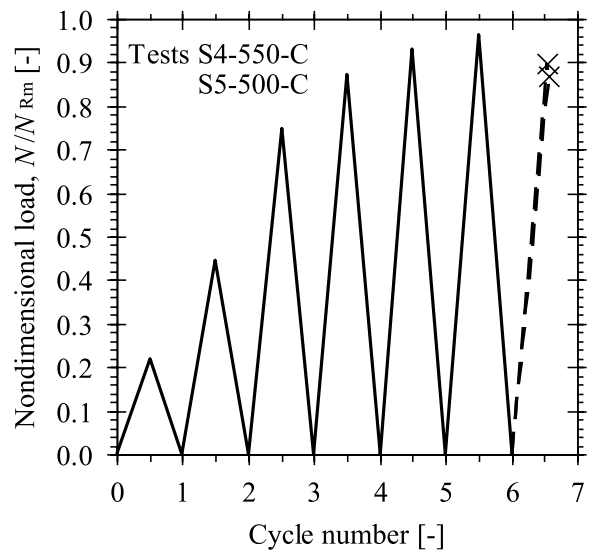

(b)

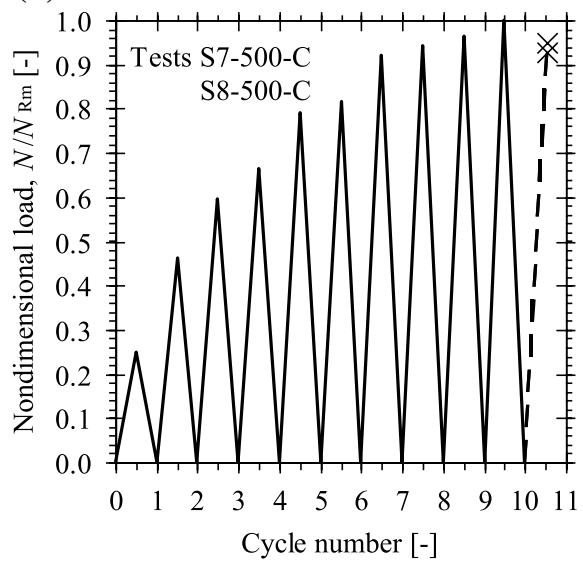

Fig. 8 Loading protocols for tests on single anchors: a tests S4-550-C and S5-500-C; b tests S7-500-C and S8-500-C

to midspan. The use of such a configuration is justified by numerical results obtained for the existing slab in the presence of earthquake loads acting in the racks transverse direction. In fact, a preliminary finite element analysis assuming the slab fixed in correspondence of 
the piles heads showed that the tensile forces at the warehouse's base, transferred to the slab by the 10 -anchor fastenings, lead to almost constant bending moment regions between the piles. These regions are long and narrow (on average $500 \mathrm{~mm}$ wide) strips aligned with the longest slab dimension.

The supports of the reaction frame were positioned at $3 \mathrm{~m}$ from one another (Fig. $7 \mathrm{~b}$ ). It is worth noting that the span length of $3 \mathrm{~m}$ coincides with the clear distance between the piles. Unconfined test conditions were ensured by a minimum distance between anchors and inner edge of the reaction frame of $2 h_{\mathrm{ef}}=1000 \mathrm{~mm}$. In fact, for these tests the reaction frame was supported at the ends on two IPE 450 profiles, having flange breadth of $190 \mathrm{~mm}$. This ensures a clear span of $3000-190=2810 \mathrm{~mm}=2 h_{\mathrm{ef}}+b_{\max }+2 h_{\mathrm{ef}}$, where $b_{\max }=810 \mathrm{~mm}$ is the maximum distance between the corner rods (see Fig. 7b).

For tests G1 and G3, the cyclic loading protocol shown in Fig. 9 was adopted. In particular, the selected ratios of the target loads of the various loading cycles to the maximum design tensile force, $N_{\mathrm{Ed}}=539 \mathrm{kN}$, were $0.1,0.3,0.6,0.8,1.0,1.5,2.0$ and 2.8 .

Test G2 was conducted on the basement damaged during test G1. Test G3 was conducted on another, initially uncracked basement.

\subsection{Test equipment and measuring system}

The reaction frame (Fig. 10a) includes two parallel, $3.5 \mathrm{~m}$-long HEB 500 profiles of steel class S275 (characteristic yield and ultimate strengths $f_{\mathrm{yk}}=275 \mathrm{MPa}$ and $f_{\mathrm{tk}}=430 \mathrm{MPa}$ ), stiffened by $10 \mathrm{~mm}$-thick steel plates at a spacing of $500 \mathrm{~mm}$. The net distance between the two profiles is of $200 \mathrm{~mm}$.

For tests on single anchors, the reaction frame was simply supported by two $172 \mathrm{~mm}$ deep steel rail profiles placed onto $5 \mathrm{~mm}$-thick, $300 \mathrm{~mm}$-wide plates (Fig. 10b). A builtup load transfer beam, made by two back-to-back UPN 220 steel profiles, was positioned transversely at midspan (Fig. 10c).

The tensile load was applied by means of a single-acting hollow-plunger hydraulic jack (Fig. 10c) with loading capacity of $326 \mathrm{kN}$ and cylinder effective area of $4660 \mathrm{~mm}^{2}$. The jack was activated by a two-speed, steel hand pump.

The oil pressure was measured using a digital pressure gauge with full scale pressure of 1380 bar and accuracy of $\pm 0.25 \%$. Taking account of the cylinder effective

Fig. 9 Loading protocol used in tests $\mathrm{G} 1$ and G3

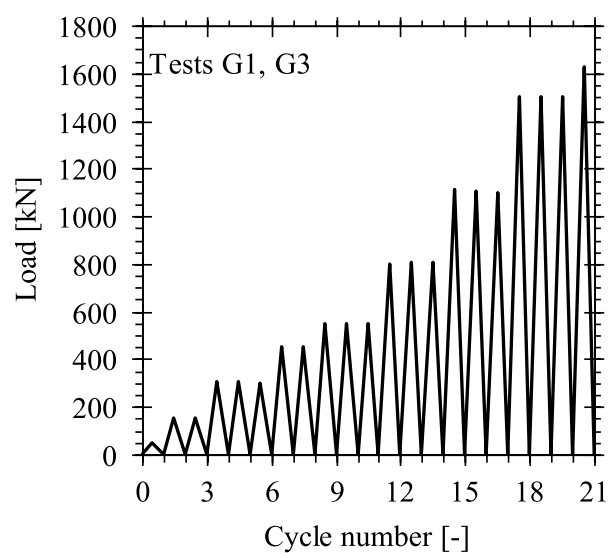



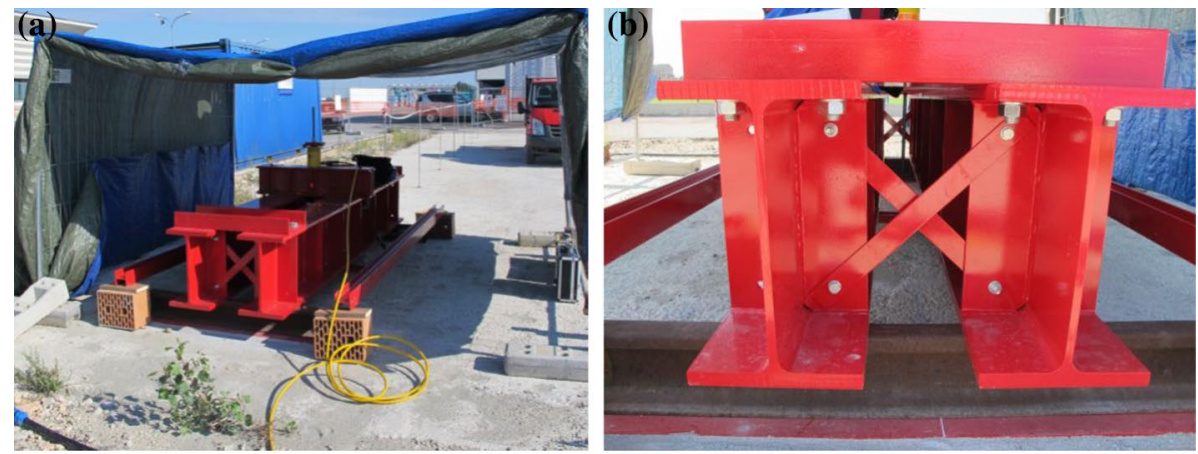

(c)
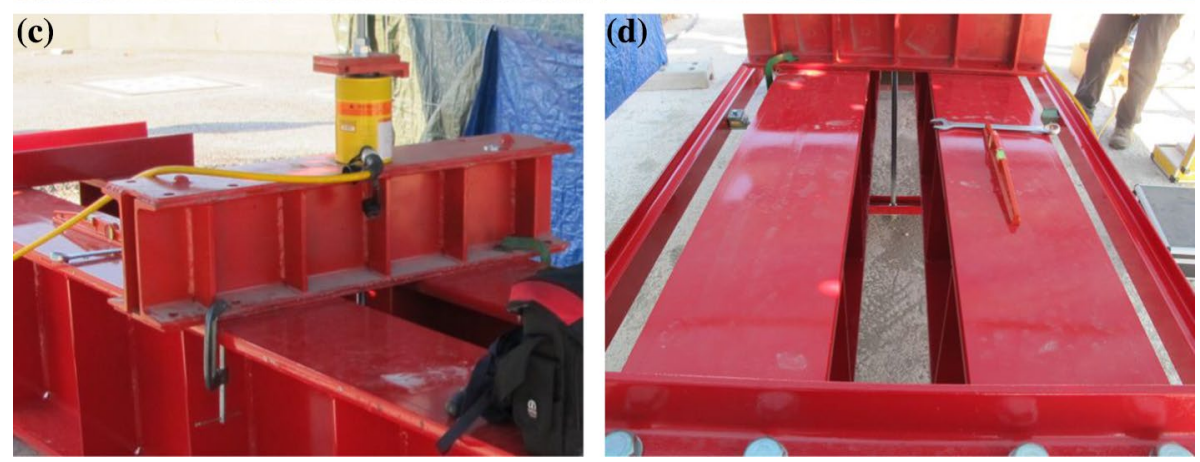

Fig. 10 Test rig for tests on single anchors: a global view; detail views showing $\mathbf{b}$ one end section of the reaction frame; $\mathbf{c}$ the actuator placed at midspan on the transverse load-transfer beam; $\mathbf{d}$ the threaded rod emerging from concrete

Section A-A

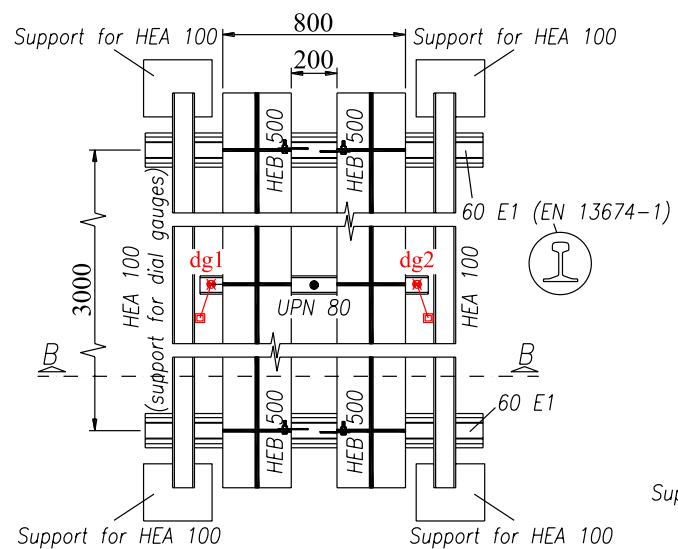

Section B-B

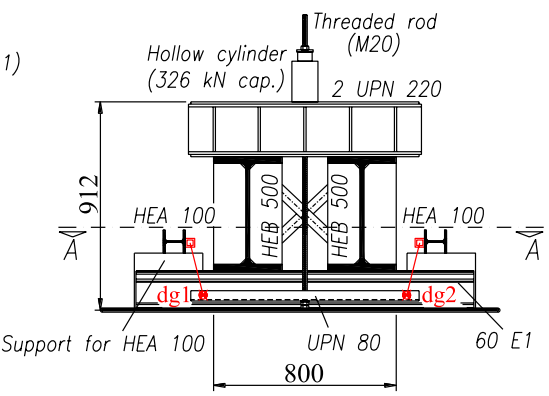

(a)

(b)

Fig. 11 a Horizontal- and b cross-sectional views of test setup showing positions of dial gauges dg1 and dg2 for tests \#1 to \#6 
area, the applied force, expressed in $\mathrm{kN}$, was obtained by multiplying the measured pressure, expressed in MPa, by factor 4.66.

In tests \#1 to \#6, the rod axial displacement was measured by two dial gauges, in the following referred to as $\operatorname{dg} 1$ and $\operatorname{dg} 2$ (Fig. 11). In order to refer the measurements to fixed points, these gauges were connected, using magnets, to metal supports independent of the reaction frame. Their touch probes were put in contact with the web of a 1 m-long UPN 80 steel profile (partially visible in Fig. 10d), positioned transversely with respect to the beams of the reaction frame. This profile, provided with a hole in centroidal position for the insertion of the threaded rod, was held by nuts at only $30 \mathrm{~mm}$ from the concrete surface. Therefore, the elongation of the rod portion emerging from concrete did not affect the displacement measurement.

In tests \#7 and \#8, the rod axial displacement was measured by gauge dg1, positioned as described above. Conversely, the touch probe of gauge dg2 was put in contact with the concrete surface at $45 \mathrm{~mm}$ from the threaded rod, in order to measure possible displacements due to uplifting of a concrete portion near the rod.

For tests on anchor groups, the reaction frame (Fig. 12a, b) was simply supported on two IPE 450 profiles directly positioned on the RC basement. Steel rolls with the diameter of $50 \mathrm{~mm}$ were interposed between the reaction frame and its supports (Fig. 12c).

The fixture (Fig. 6b) includes a ribbed steel plate of in-plane dimensions $820 \times 890 \mathrm{~mm}$ and two $30 \mathrm{~mm}$-thick vertical plates provided with a $72 \mathrm{~mm}$-diameter hole. Plan views of one of the basements with the fixture and the reaction frame are shown in Fig. 13a, b, respectively. The tensile load was applied to the fixture by means of a 47 mm-diameter Dywidag bar (Fig. 13c, e). In particular, a threaded sleeve suitable for this bar was welded to a $60 \mathrm{~mm}$-thick vertical plate, also provided with a $72 \mathrm{~mm}$-diameter hole. The assemblage including Dywidag bar, sleeve and plate was then inserted into the space between the two vertical plates of the fixture (Figs. 6b, $13 \mathrm{c}, \mathrm{e})$, and pinned using a $70 \mathrm{~mm}$-diameter pin. At the top end, the Dywidag bar was tightened to a ribbed steel lid (Fig. 13d), on which the plungers of two identical hydraulic jacks were acting (Figs. 12a, b, 13c, e). Each of these jacks was a doubleacting cylinder with loading capacity of $1 \mathrm{MN}$ and advance effective area of 13,330 $\mathrm{mm}^{2}$. The jacks were connected in parallel to the same electrical pump.

The oil pressure was measured using the same digital gauge already used for tests on single anchors. Taking account of the advance effective area of the two cylinders, the applied force, expressed in $\mathrm{kN}$, was obtained by multiplying the measured pressure, expressed in $\mathrm{MPa}$, by factor 26.66 .

The absolute vertical displacement of the fixture was measured in four points by dial gauges dg1 to dg4 (Fig. 14). These gauges were connected with magnets to $3.5 \mathrm{~m}$-long HEA 100 beams independent of the reaction frame. In test G1, the HEA 100 profiles were aligned orthogonally to the beams of the reaction frame (Fig. 14a) and the displacement measurements were affected by the flexural deformation experienced by the basement during the test (see Sect. 4.3). Therefore, in tests G2 and G3 these beams were aligned parallel to the HEB 500 beams of the reaction frame (Fig. 14b) and supported at zones of the basements not subjected to uplift.

In all of the tests, the vertical displacement of the fixture relative to concrete was measured by dial gauges dg5 and dg6 (Fig. 14). These gauges were connected with magnets to the fixture and their touch probes were put in contact with the concrete surface. 

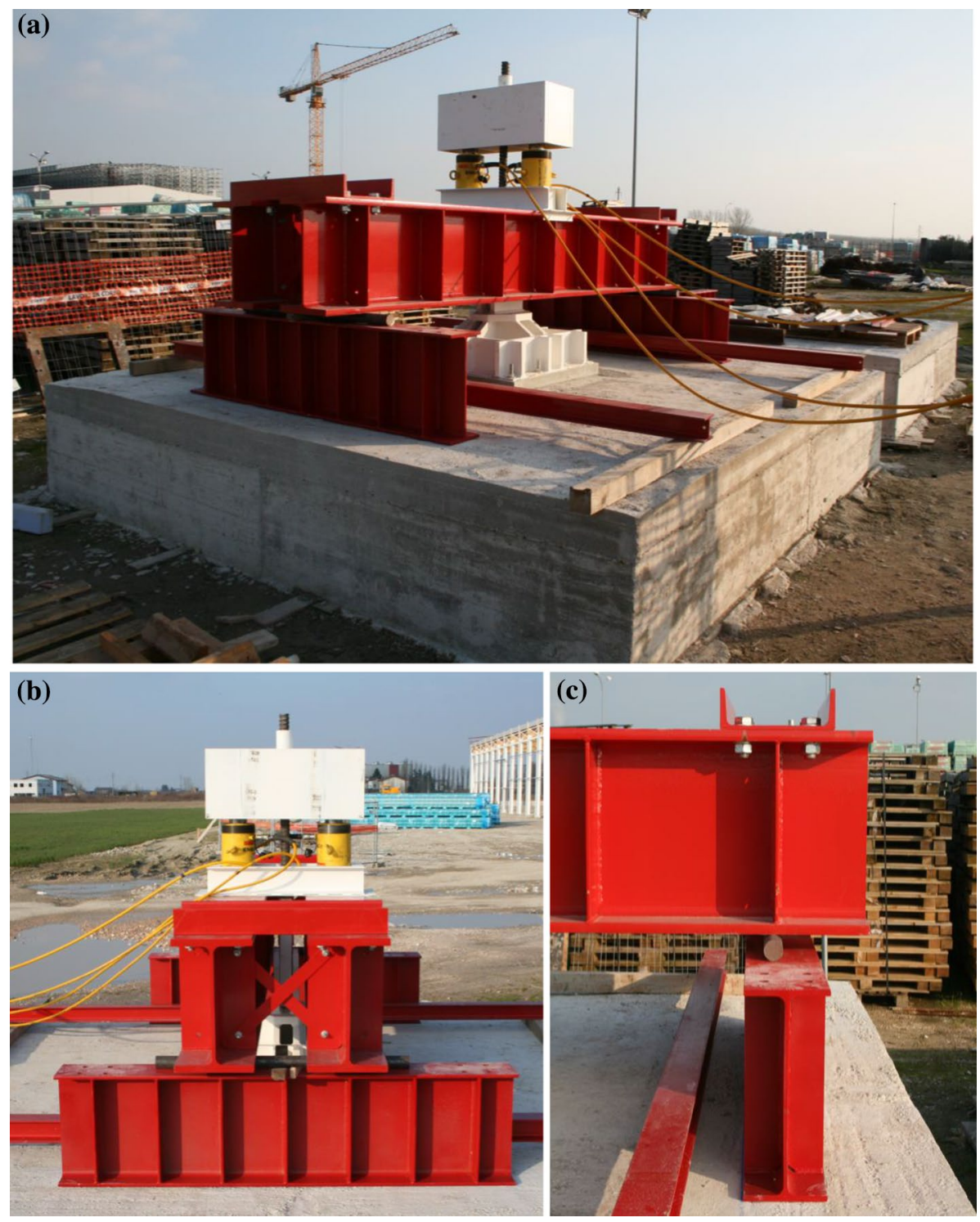

Fig. 12 Setup for tests on anchor groups: a overall view; b front view of test rig; $\mathbf{c}$ detail view of one of the supports of the reaction frame

\section{Experimental results}

The main results of tests on single anchors and anchor groups are presented in Sects. 4.1 and 4.2 , respectively. 

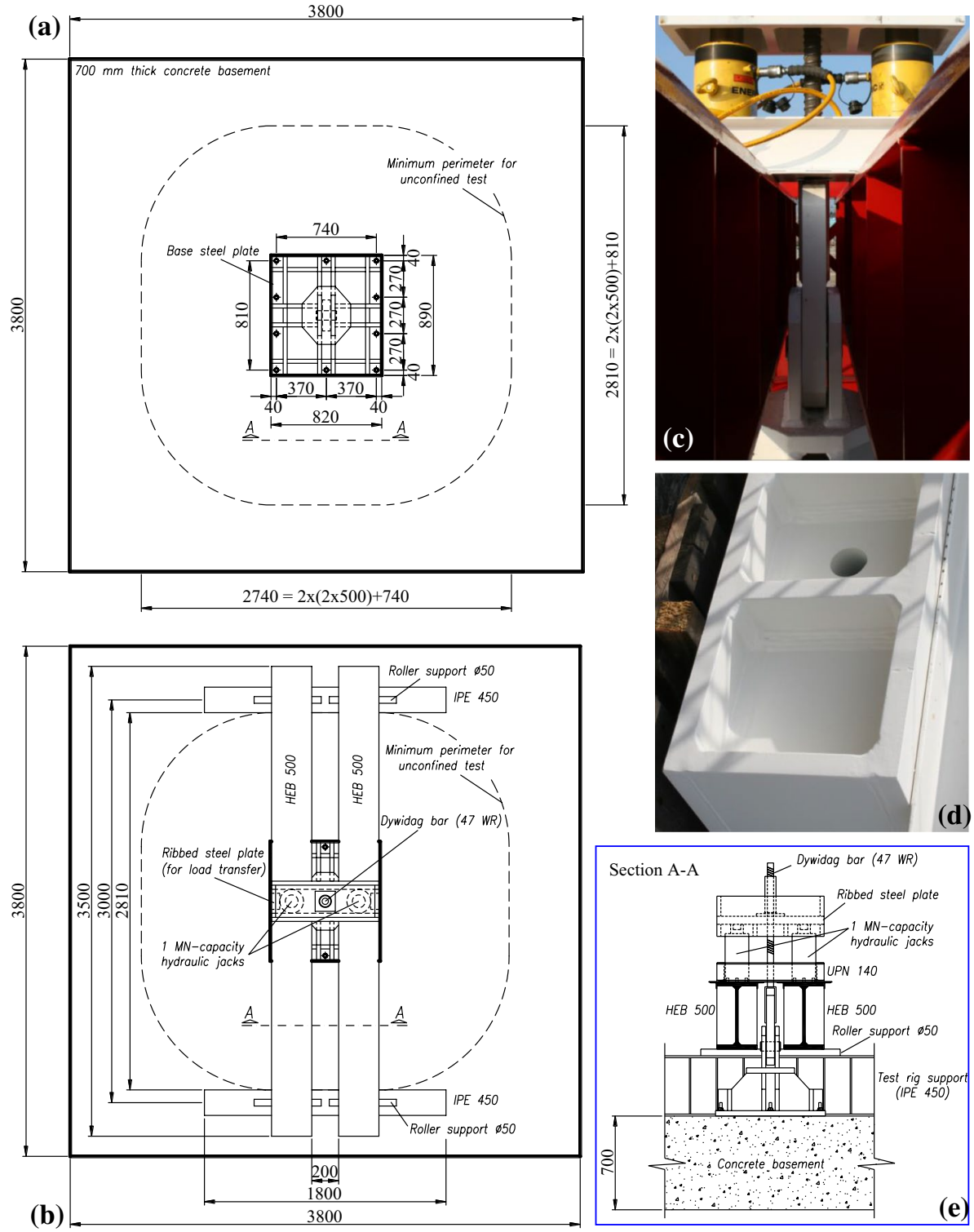

Fig. 13 Tests on anchor groups: in-plane dimensions of a RC basement with the a installed fastening and $\mathbf{b}$ reaction frame; detail view of $\mathbf{c}$ system for anchor tensioning and $\mathbf{d}$ ribbed steel lid; e cross-sectional view of test setup

\subsection{Single anchors}

Monotonic tests \#1 and \#3 were stopped at the achievement of the actuator stroke length, with the rod resisting a force (see Table 1) about $2 \%$ greater than the mean tensile resistance reported in Table 2. In all other tests, the rod failure (mode $S$ in Table 1) was attained (see Fig. 15a). Concrete cracking observed at the end of tests was more pronounced in the case 
(a)

Direction of

HEB 500 beams

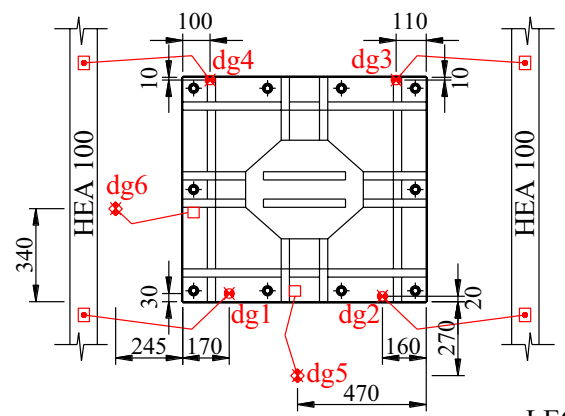

Control station (b)

Direction of

HEB 500 beams
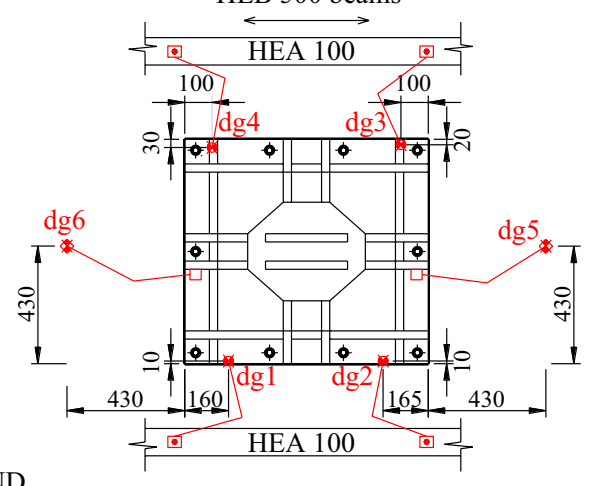

LEGEND

* Dial gauge

Control station

o. Magnetic stand

Fig. 14 Positions of dial gauges during tests a G1 and b G2 and G3
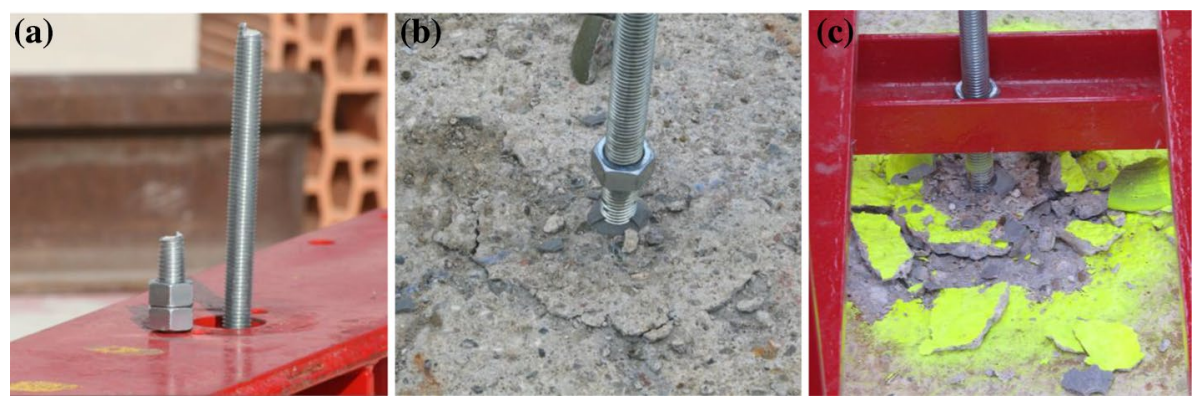

Fig. 15 Failure modes observed in tests on single anchors: a rod failure (test S2-550-M); concrete cracking at up to b $80 \mathrm{~mm}$ (test S5-500-C) and c 150-200 mm (test S8-500-C) from the rod

of cyclic loading. In particular, almost circular cracks with a diameter of approximately $160 \mathrm{~mm}$, centred around the rod, were observed in tests S4-550-C (\#4) and S5-500-C (\#5), see Fig. 15b. In tests S7-500-C (\#7) and S8-500-C (\#8) the cracks involved a more extended region of the concrete surface, having diameter of 300-400 mm (Fig. 15c), probably because of the greater number of loading cycles performed (see Fig. 8).

The load-mean displacement plots obtained from tests S4-550-C (\#4) and S5-500-C (\#5) are reported in Fig. 16a, b, respectively, where $u_{\mathrm{dg} i}(i=1,2)$ stands for displacement measured by gauge dgi and cross symbol indicates the failure point. The ultimate conditions, governed by steel failure, were attained at substantially coincident tensile loads and the difference in the embedment depths adopted in the two tests did not play any significant role.

The load-displacement plots obtained from all of the tests are compared in Fig. 16c. In particular, for cyclic tests the envelope curve of the cyclic load-displacement diagram is reported in the figure. Moreover, the displacement values in the figure coincide with $\left(u_{\mathrm{dg} 1}+u_{\mathrm{dg} 2}\right) / 2$ for tests \#1 to \#6 and with $u_{\mathrm{dg} 1}$ for tests \#7 and \#8. Also reported in the figure 
(a)

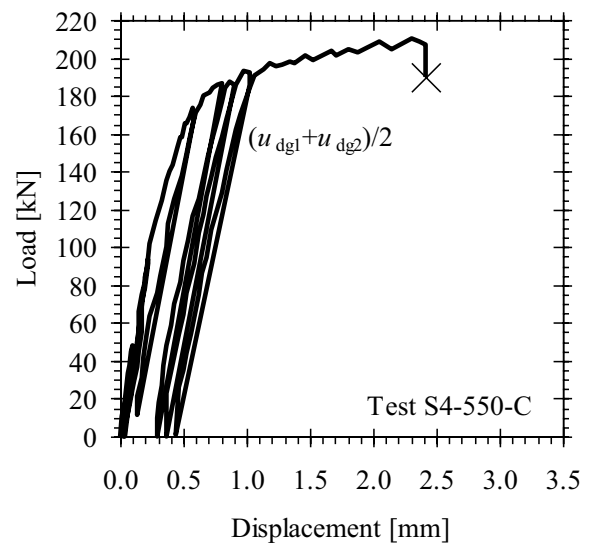

(b)

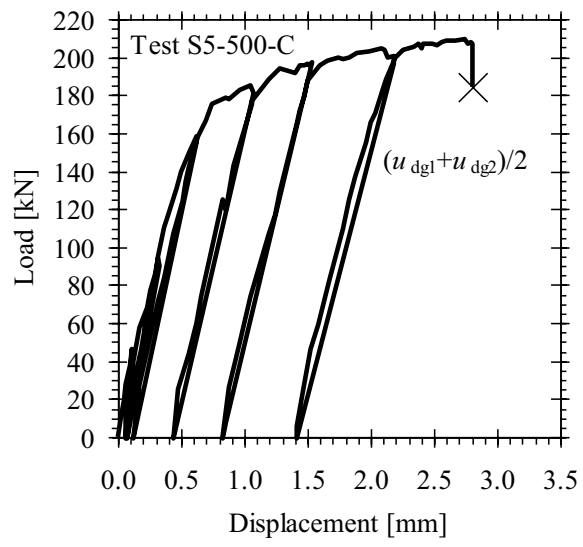

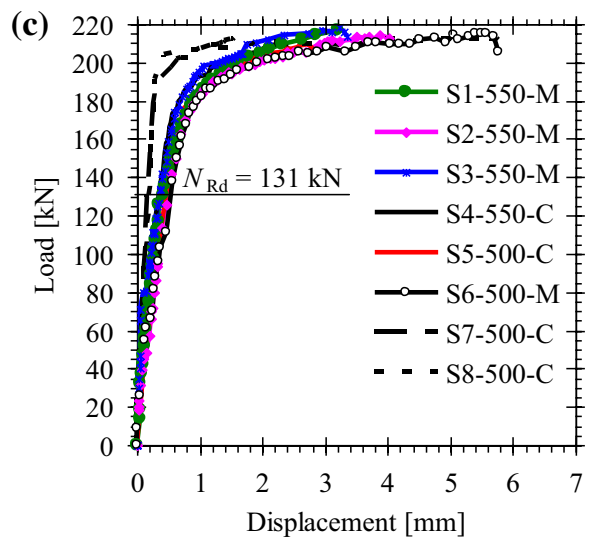

Fig. 16 Tests on single anchors: cyclic load-mean displacement responses obtained from tests a S4-550-C and b S5-500-C; c load-displacement plots for all tests (envelopes for cyclic tests)

is a horizontal line at $N_{\mathrm{Rd}}=N_{\mathrm{Rd}, \mathrm{s}}=N_{\mathrm{Rk}, \mathrm{s}} / \gamma_{\mathrm{Ms}}=196 / 1.5=131 \mathrm{kN}$, corresponding to design resistance of steel rods (see Sect. 5.1). The curves for the first six tests are quite close to one another. Those obtained from the last two tests show a higher elastic stiffness, probably with an underestimate, in the elastic range, of the rod displacement due to the use of one single gauge.

Peak loads and rod maximum displacements are summarized in Table 1. For tests \#7 and \#8, quantity $u_{\mathrm{dg} 1}-u_{\mathrm{dg} 2}$ provides an estimate of the slip experienced by the rod with respect to concrete. The greatest rod displacement and slip were obtained in test \#8 and resulted to be $5.83 \mathrm{~mm}$ and $3.08 \mathrm{~mm}$, respectively. The mean value of the rod maximum displacement obtained from monotonic tests, approximately equal to $4 \mathrm{~mm}$, is $27 \%$ greater than that obtained from cyclic tests $(3.2 \mathrm{~mm})$.

The mean value and standard deviation of the peak tensile resistance are reported in Table 3 for the tests grouped according to different rules. The mean resistance is in line with the value of $N_{\mathrm{Rm}}$ reported in Table 2 and the maximum standard deviation resulted to be approximately equal to $3 \%$. 
Table 3 Mean values and standard deviations of the tensile capacities obtained from tests on single anchors

\begin{tabular}{lll}
\hline Tests on single anchors & Peak load \\
\cline { 2 - 3 } & Mean value (kN) & SD (kN) \\
\hline Monotonic (tests \#1, \#2, \#3 and \#6) & 216.0 & 1.2 \\
Cyclic (tests \#4, \#5, \#7 and \#8) & 211.3 & 2.0 \\
$h_{\text {ef }}=550 \mathrm{~mm}$ (tests \#1 to \#4) & 214.4 & 3.1 \\
$h_{\text {ef }}=500 \mathrm{~mm}$ (tests \#5 to \#8) & 212.9 & 3.0 \\
All tests & 213.7 & 2.9 \\
\hline
\end{tabular}

\subsection{Anchor groups}

In test G1, cracks on the basement top surface were first observed during loading cycle \#18 for a total tensile force of approximately $1550 \mathrm{kN}$. These cracks were mainly oriented orthogonally to the HEB 500 beams of the reaction frame (Fig. 17a, b), indicating a typical flexural failure mode. Other cracks originated diagonally (Fig. 17c) from two of the corners of the fixture. A plan view of the top surface is shown in Fig. 18a, b, where the crack pattern at the end of test is highlighted in blue. It is worth noting that the cracking was not restricted to the top surface, but involved also the vertical faces of the basement parallel to the reaction frame (Fig. 17a, b). At the maximum load reached during the test, $1627 \mathrm{kN}$

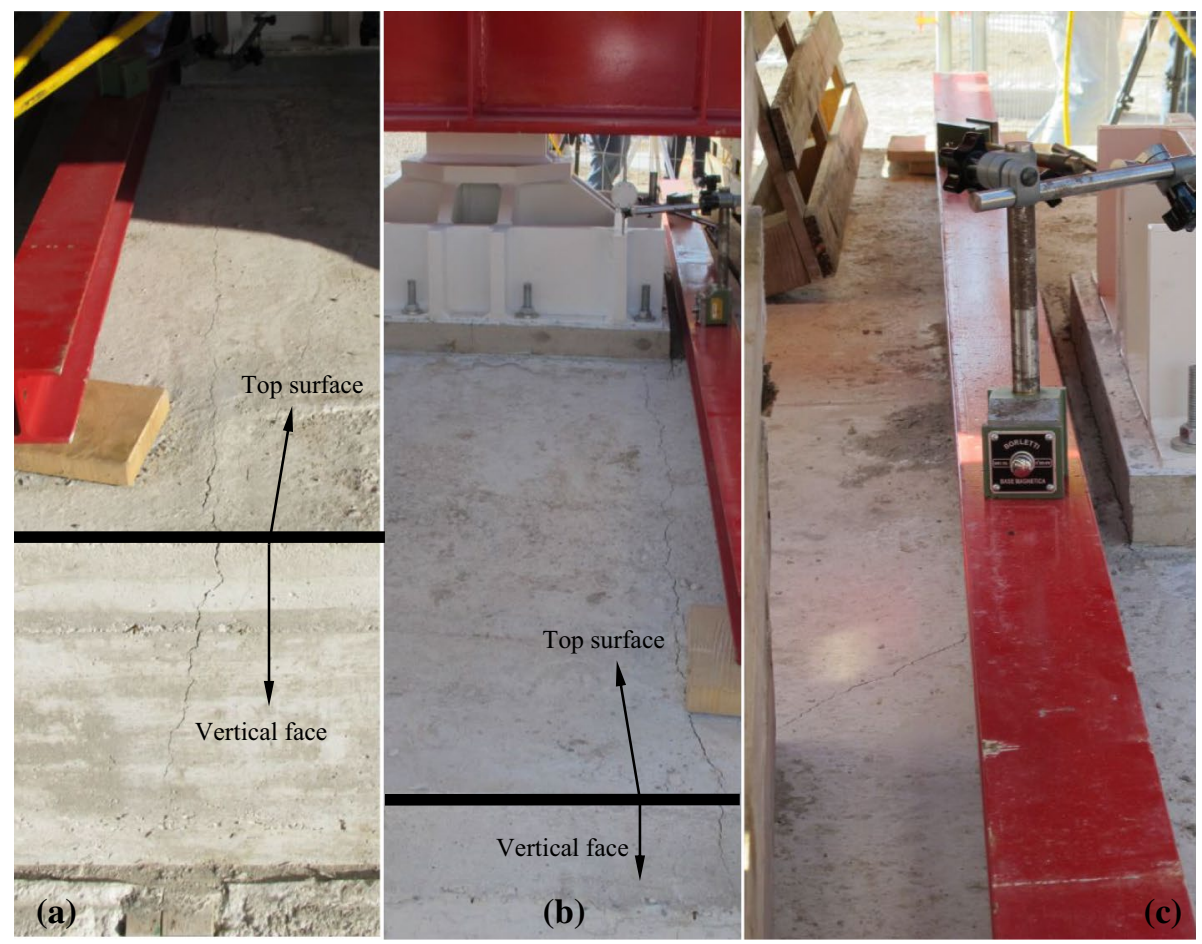

Fig. 17 Crack formation during test G1: a, b cracks orthogonal to the beams of the reaction frame; c diagonal crack 

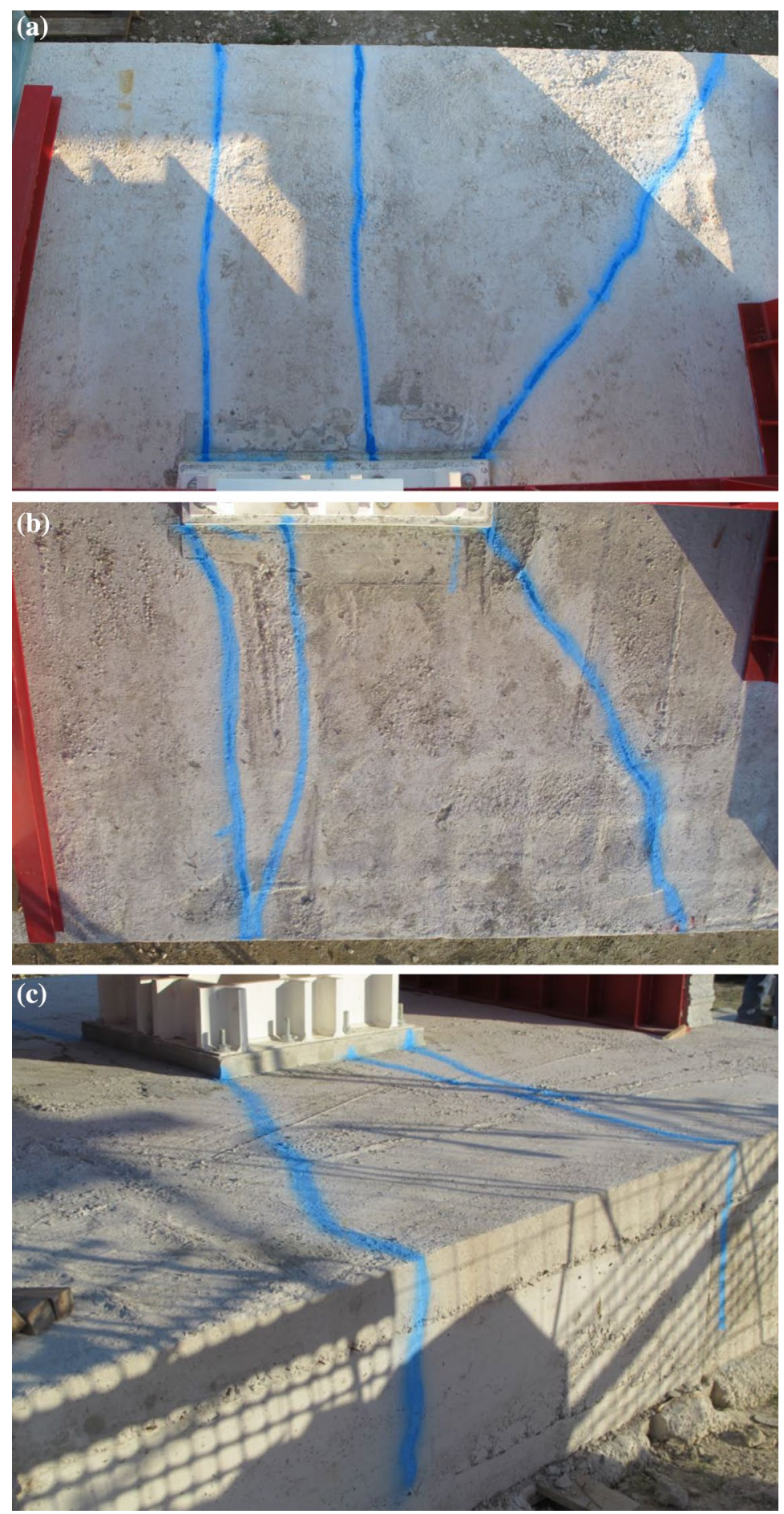

Fig. 18 Crack patterns (highlighted in blue) in RC basements at the end of tests $\mathbf{a}, \mathbf{b}$ G1 and $\mathbf{c}$ G3 
(Table 1), the neutral axis depth at midspan, based on the measure of the uncracked depth of the basement, was of approximately $80 \mathrm{~mm}$.

The cyclic diagram of total applied load versus mean displacement is reported in Fig. 19a. The mean displacement is computed as $\Sigma_{i} u_{\mathrm{dg} i} / 4$, with $u_{\mathrm{dg} i}(i=1, \ldots, 4)$ being the displacement measured by dial gauge dgi. The envelope curve of the cyclic diagram is also depicted in the figure (red solid line) and clearly shows a sort of "yield plateau" for the load of $1500 \mathrm{kN}$, followed by a branch of stiffness smaller than the initial one. In the same figure, the red dashed line represents the envelope curve of the cyclic diagram of total load versus fixture relative displacement. This displacement is computed as $\left(u_{\mathrm{dg} 5}+u_{\mathrm{dg} 6}\right) / 2$, with $u_{\mathrm{dg} i}(i=5,6)$ being the measurement of relative displacement between fixture and concrete. The two envelopes are quite close to one another, because measurements $u_{\mathrm{dg} 1}$ to $u_{\mathrm{dg} 4}$ were affected by rigid vertical displacements experienced by the gauges supports (see Sect. 4.2), resulting in an underestimate of the fixture absolute displacement.

The objective of test G2, conducted on the same basement as test G1, was therefore to avoid such an underestimate adopting a different arrangement of the HEA 100 beams used

(a)

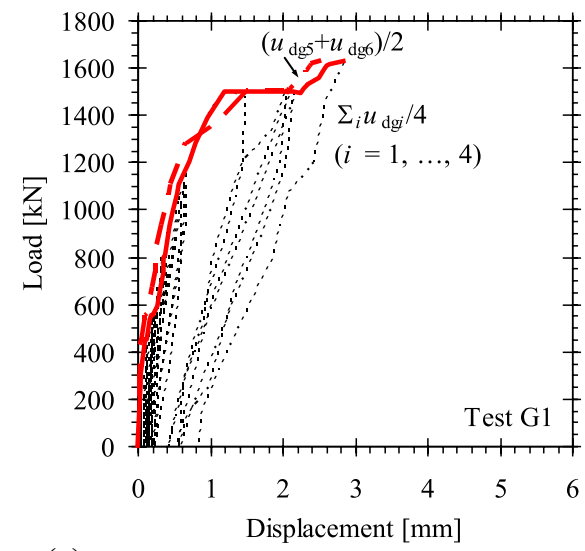

(c)

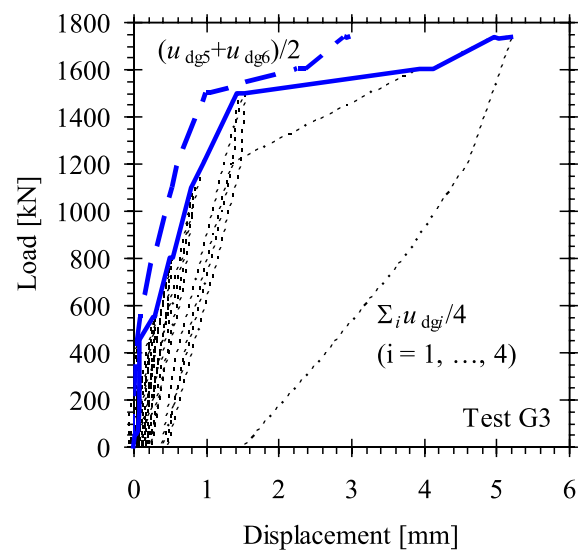

(b)

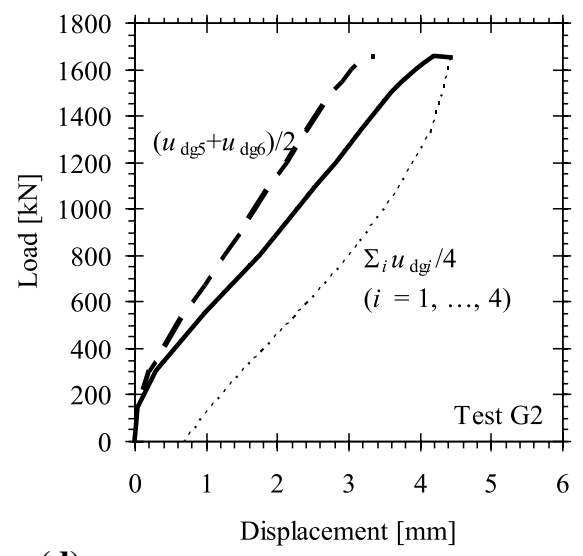

(d)

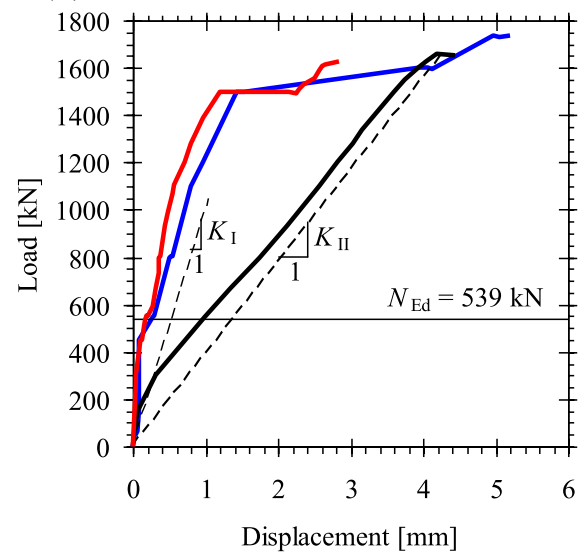

Fig. 19 Tests on anchor groups: load-mean displacement responses and envelopes obtained from tests a G1, b $\mathrm{G} 2$ and $\mathbf{c} \mathrm{G} 3$; $\mathbf{d}$ comparison between the envelopes 
Table 4 Coefficients used in Table 5.

\begin{tabular}{|c|c|c|c|}
\hline \multicolumn{2}{|c|}{ Symbol, description (Unit) } & Equation/figure & Value \\
\hline \multicolumn{4}{|c|}{ Common quantities } \\
\hline$s_{1}, s_{2}$ & Anchor spacings (mm, mm) & Fig. $13 \mathrm{a}$ & 270,370 \\
\hline$k_{1}$ & Coefficient for uncracked concrete $(-)$ & & 11 \\
\hline \multicolumn{4}{|c|}{ Quantities related with Mode P failure } \\
\hline$n$ & Number of anchors in the group (-) & Figs. 13a, 21 & 10 \\
\hline$s$ & Average spacing $(\mathrm{mm})$ & $s=\left(3 s_{1}+2 s_{2}\right) / 5$ & 310 \\
\hline$s_{\mathrm{cr}, N \mathrm{p}}$ & Critical spacing (mm) & Equation (18) & 462 \\
\hline$A_{\mathrm{p}, N}^{0}$ & Reference area of an individual anchor $\left(\mathrm{mm}^{2}\right)$ & $A_{\mathrm{p}, N}^{0}=s_{\mathrm{cr}, N \mathrm{p}}^{2}$ & $2.13 \times 10^{5}$ \\
\hline$A_{\mathrm{p}, N}$ & Actual bond influence area $\left(\mathrm{mm}^{2}\right)$ & $A_{\mathrm{p}, N}=s_{\mathrm{cr}, N \mathrm{p}}\left(6 s_{1}+4 s_{2}\right)$ & $1.43 \times 10^{6}$ \\
\hline \multicolumn{4}{|c|}{ Quantities related with Mode C failure } \\
\hline$s_{\mathrm{cr}, N}$ & Critical spacing (mm) & $3 h_{\mathrm{ef}}$ & 1500 \\
\hline$A_{\mathrm{c}, N}^{0}$ & Reference projected area for one anchor $\left(\mathrm{mm}^{2}\right)$ & $A_{\mathrm{c}, N}^{0}=9 h_{\mathrm{ef}}^{2}$ & $2.25 \times 10^{6}$ \\
\hline$A_{\mathrm{c}, N}$ & Actual projected area $\left(\mathrm{mm}^{2}\right)$ & $A_{\mathrm{c}, N}=\left(3 s_{1}+s_{\mathrm{cr}, N}\right)\left(2 s_{2}+s_{\mathrm{cr}, N}\right)$ & $5.17 \times 10^{6}$ \\
\hline
\end{tabular}

Reference and actual areas were calculated for $h_{\mathrm{ef}}=500 \mathrm{~mm}$

to support the dial gauges. The maximum load reached during the test was $2 \%$ larger than that reached in test G1 (Table 1), whereas the measured maximum total displacement of the fixture resulted $56 \%$ greater. The load-absolute displacement plot for test G2 is reported in Fig. 19b (solid line for the ascending branch). The stiffness of this curve coincides with that of the final branch of the envelope curve reported with red solid line in Fig. 19a. The dashed line in Fig. 19b refers to relative displacements between fixture and concrete.

In test G3, a maximum load about $7 \%$ larger than in test G1 was attained (Table 1). It was decided not to increase further the applied load in order not to yield the Dywidag bar of the tensioning system. Anyway, the ratio of the maximum experimental load to the computed design load, $N_{\text {Ed }}$ (see Sect. 2), is $1736 / 539=3.2$. The observed crack pattern (Fig. 18c), involving both top surface and front and back sides of the basement, was analogous to that observed in test G1, but the measured neutral axis depth at midspan was approximately equal to $50 \mathrm{~mm}$. The cyclic and envelope load-displacement plots are reported in Fig. 19c and show a strong stiffness reduction for a total load of $1500 \mathrm{kN}$. Then, the specimen experienced a significant increase of the vertical displacement up to a load of $1600 \mathrm{kN}$, corresponding to crack formation, followed by a branch of stiffness even smaller than that observed in test G2 (see Fig. 19d).

The dashed lines with slopes $K_{\mathrm{I}}$ and $K_{\mathrm{II}}$ reported in Fig. 19d represent the linear elastic load-displacement responses for uncracked and cracked concrete, respectively, estimated on the basis of the experimental results. These stiffnesses, taking account of the slip between threaded rods and surrounding concrete, result to be $K_{\mathrm{I}}=1000 \mathrm{kN} / \mathrm{mm}$ and $K_{\mathrm{II}}=390 \mathrm{kN} / \mathrm{mm}$.

\section{Analitical interpretation}

The failure loads for fastenings with post-installed bonded anchors are evaluated analytically in this Section to interpret the experimental results. For the detailed calculations, according to CEN (2018), of the failure loads corresponding to $h_{\mathrm{ef}}=500$ and $550 \mathrm{~mm}$ in the case of single 
anchors and to $h_{\mathrm{ef}}=500 \mathrm{~mm}$ in the case of anchor groups, the reader should refer to Tables 4 and 5. In particular, mean (index m), characteristic (index k) and design (index d) failure loads are given in Table 5 .

\subsection{Failure loads for single anchors}

To interpret the experimental results for single anchors all possible failure modes were examined adopting mean values of material properties. Due to the distances between anchors and edges of the foundation slab adopted in the tests, the influence of edge distances was not accounted for in the calculations. The failure loads are plotted in Fig. 20a versus $h_{\mathrm{ef}} / d$ lying between 0 and 35 (corresponding to $h_{\mathrm{ef}}=h=700 \mathrm{~mm}$, i.e., the thickness of the foundation slab).

With regard to concrete-related failure modes, pullout failure load (Mode P) was estimated from the following equation (CEN 2018, Eq. (7.14) with coefficient $\psi_{\text {sus }}=1$ ):

$$
N_{\mathrm{Rm}, \mathrm{p}}=\tau_{\mathrm{Rm}} \pi d h_{\mathrm{ef}},
$$

Table 5 Calculations of the failure loads for Modes P, C and S for the fastenings tested in this research

\begin{tabular}{lllll}
\hline Symbol, description (Unit) & Equation & & \multicolumn{2}{l}{ Value } \\
\cline { 3 - 5 } & & $(.)_{\mathrm{m}}$ & $(.)_{\mathrm{k}}$ & $(.)_{\mathrm{d}}$ \\
\hline
\end{tabular}

Failure loads for single anchors

Mode $P$

$N_{\mathrm{R}, \mathrm{p}} \quad$ Failure load for one anchor $[\mathrm{kN}]$

Eq. (9)

$h_{\text {ef }}=500 \mathrm{~mm}$

397

339

188

$h_{\mathrm{ef}}=550 \mathrm{~mm}$

Mode C

$N_{\mathrm{R}, \mathrm{c}} \quad$ Failure load for one anchor $(\mathrm{kN})$

Eq. (10)

$$
\begin{aligned}
& h_{\text {ef }}=500 \mathrm{~mm} \\
& h_{\mathrm{ef}}=550 \mathrm{~mm}
\end{aligned}
$$

$\begin{array}{lll}758 & 674 & 374 \\ 875 & 777 & 432\end{array}$

Mode $S$

$N_{\mathrm{R}, \mathrm{s}} \quad$ Failure load for one anchor $(\mathrm{kN})$

$212 \quad 196$

Failure loads for anchor groups $\left(h_{\mathrm{ef}}=500 \mathrm{~mm}\right)$

Mode $P$

$\tau_{\mathrm{R}, \max }$

$\psi_{\mathrm{g}, N \mathrm{p}}^{0}$

Maximum bond strength (MPa)

$\psi_{\mathrm{g}, N \mathrm{p}}$

$N_{\mathrm{R}, \mathrm{p}}^{0}$

$N_{\mathrm{R}, \mathrm{p}}$

Factor accounting for $\tau_{\mathrm{R}}(-)$

Equation (17)

21.2

19.6

Equation (16)

2.29

2.32

Equation (15)

1.23

1.24

Failure load for one anchor $(\mathrm{kN})$

Equation (9)

364

Equation (14)

3012

327

Failure load for the anchor group $(\mathrm{kN})$

Equation (10)

667

Equation (19)

1533

615

Failure load for the anchor group $(\mathrm{kN})$

Equation (19)

1414

Mode $S$

$N_{\mathrm{R}, \mathrm{s}}^{0} \quad$ Failure load for one anchor $(\mathrm{kN})$

$212 \quad 196$

$N_{\mathrm{R}, \mathrm{s}}$

Failure load for the anchor group $(\mathrm{kN})$

$n N_{\mathrm{R}, \mathrm{s}}^{0}$

2120

1960 
(a)
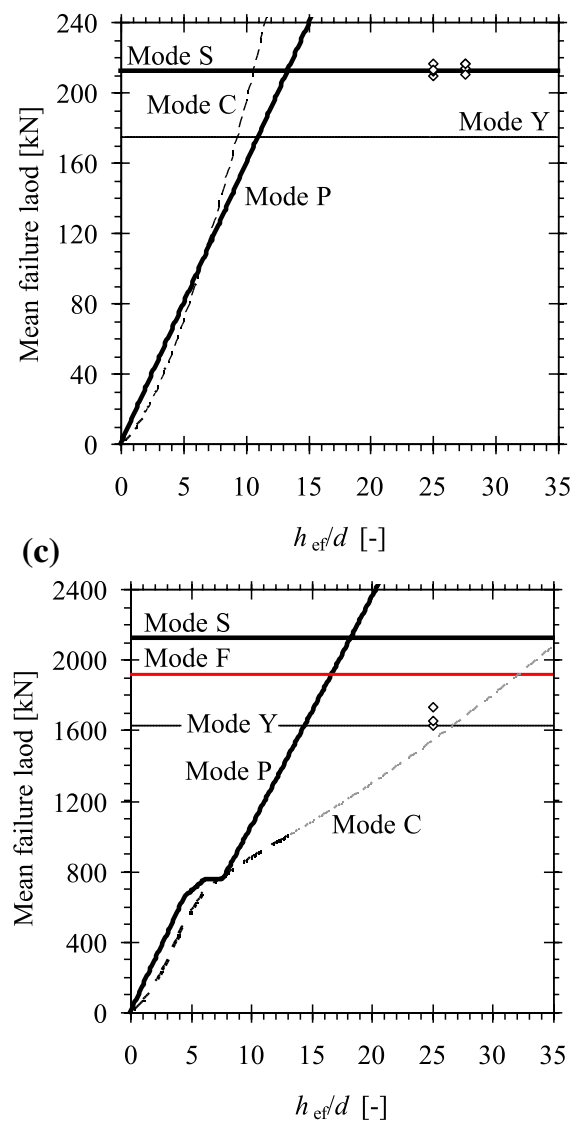

(b)
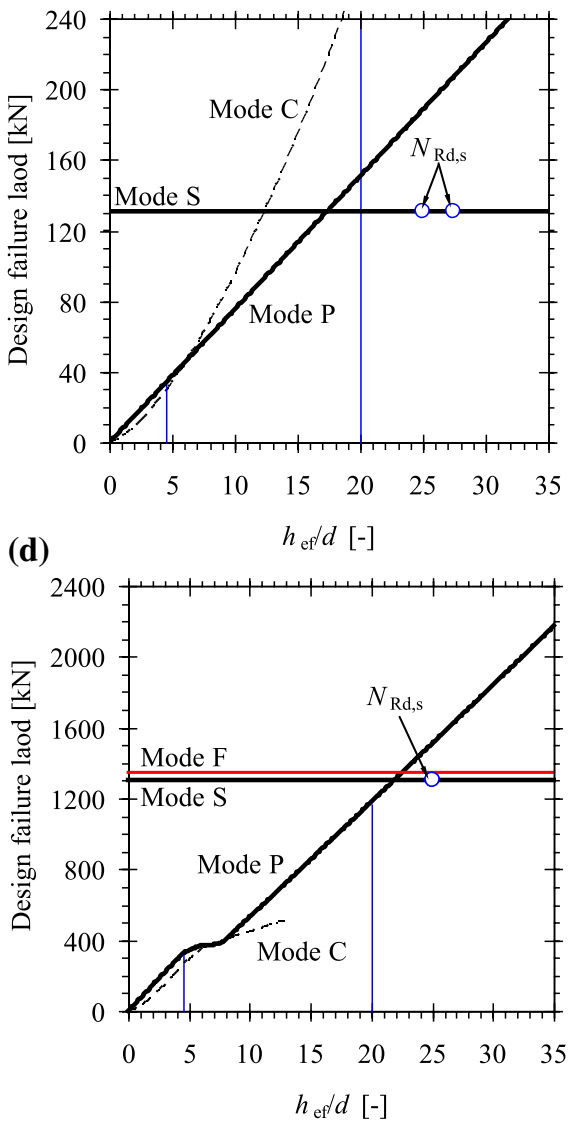

Fig. 20 Comparison between predicted failure loads for $\mathbf{a}, \mathbf{b}$ single anchors and $\mathbf{c}, \mathbf{d}$ anchor groups. Mean and design material properties were used in $\mathbf{a}, \mathbf{c}$ and $\mathbf{b}, \mathbf{d}$, respectively. Diamond data points in $\mathbf{a}, \mathbf{c}$ locate the experimental failure loads. Vertical blue lines in $\mathbf{b}, \mathbf{d}$ identify the $h_{\mathrm{ef}}$ range of application specified by FIB (2011)

whereas concrete cone failure load (Mode C) was obtained from (CEN 2018, Eq. (7.2)):

$$
N_{\mathrm{Rm}, \mathrm{c}}=k_{1} \sqrt{f_{\mathrm{cm}}} h_{\mathrm{ef}}^{1.5},
$$

with $f_{\mathrm{cm}}$ and $\tau_{\mathrm{Rm}}$ reported in Table 2 . In particular, the value $\tau_{\mathrm{Rm}}=\tau_{\mathrm{Rm} \text {,ucr }}$ was used in Eq. (2). Since only the characteristic value of the bond strength was provided by the manufacturer, $\tau_{\mathrm{Rm}}$ was approximated based on the following relation:

$$
\tau_{\mathrm{Rm}}=\tau_{\mathrm{Rk}} f_{\mathrm{cm}}^{2 / 3} / f_{\mathrm{ck}}^{2 / 3},
$$

where proportionality between bond strength and $f_{\mathrm{c}}^{2 / 3}$ was borrowed by CEN (2004). For uncracked concrete, coefficient $k_{1}$ appearing in Eq. (3) is given by $k_{1}=k_{\mathrm{ucr}, N}=11$. In estimating, with Eq. (2), the failure load corresponding to Mode P, a uniform bond stress distribution was assumed along the embedment depth also for $h_{\mathrm{ef}}$ larger than the upper bound for bonded anchors $(=20 d)$ set by CEN (2018). 
The steel failure load (Mode S) was assumed to coincide with $N_{\mathrm{Rm}, \mathrm{s}}$ reported in Table 5. In analogy with the calculation of the development length for reinforcing bars, usually based on the yield strength, the load corresponding to yielding of the threaded rod (Mode Y) is also introduced in Fig. 20a for comparison purposes (see Mahrenholtz et al. 2015). This load was estimated from the change in slope of the load-mean displacement plots obtained from single anchor tests (Fig. 16), resulting in $N_{\mathrm{ym}}=175 \mathrm{kN}$.

Also reported in Fig. 20a are the experimental failure loads, which are very well approximated by Mode S. In fact, the embedment depths of the tested anchors were significantly greater than $14 d$, i.e., the value corresponding to the intersection between Mode $\mathrm{P}$ and Mode $\mathrm{S}$, and were therefore large enough to prevent any concrete-related failure mode.

The design failure loads corresponding to the same modes described above (with the exception of Mode Y) are reported, once again versus $h_{\mathrm{ef}} / d$, in Fig. 20b. With regard to concrete-related failure modes, Eqs. (2) and (3) still were used, but with $f_{\mathrm{ck}}$ and $\tau_{\mathrm{Rk}}=\tau_{\mathrm{Rk} \text {,ucr }}$ (see Table 2) in the place of $f_{\mathrm{cm}}$ and $\tau_{\mathrm{Rm}}$, respectively. Moreover, the resulting failure loads were divided by partial safety factor (CEN 2018) $\gamma_{\mathrm{Mp}}=\gamma_{\mathrm{Mc}}=\gamma_{\mathrm{c}} \gamma_{\text {inst }}=1.8$, with $\gamma_{\mathrm{c}}=1.5$ and $\gamma_{\text {inst }}=1.2$ (normal installation safety). In the case of steel failure, the design resistance was obtained from the following relation:

$$
N_{\mathrm{Rd}, \mathrm{s}}=N_{\mathrm{Rk}, \mathrm{s}} / \gamma_{\mathrm{Ms}}=A_{\mathrm{res}} f_{\mathrm{yk}} / \gamma_{\mathrm{Ms}}
$$

where, for $20 \mathrm{~mm}$-diameter threaded rods, $A_{\text {res }}=245 \mathrm{~mm}^{2}$. The partial safety factor was obtained from the equation (CEN 2018):

$$
\gamma_{\mathrm{Ms}}=1.2 f_{\mathrm{uk}} / f_{\mathrm{yk}} \geq 1.4
$$

In Eqs. (5) and (6), $f_{\mathrm{uk}}$ and $f_{\mathrm{yk}}$ are the characteristic ultimate and yield strengths, respectively. For threaded rods of class 8.8 (IMIT 2018), $f_{\mathrm{uk}}=800 \mathrm{MPa}$ and $f_{\mathrm{yk}}=640 \mathrm{MPa}$, leading to $\gamma_{\mathrm{Ms}}=1.5$.

In Fig. 20b, the failure load for the tested anchors is located by circle data points for $h_{\mathrm{ef}} d d=25$ and 27.5. Vertical blue lines in Fig. 20b locate the range of $h_{\mathrm{ef}}$ values recommended by the manufacturer to comply with the European standard (CEN 2018), i.e., $4.5 d \leq h_{\mathrm{ef}} \leq 20 d$. The intersection between failure load curves for Mode $\mathrm{P}$ and Mode $\mathrm{S}$, lying within this range $\left(h_{\mathrm{ef}} / d=17.5\right)$, indicates that adopting $h_{\mathrm{ef}}=350 \mathrm{~mm}$ is sufficient to obtain the same design failure load as that for the tested anchors.

\subsection{Failure loads for anchor groups}

The failure modes predicted, using mean values of material properties, for the anchor groups investigated in this paper are compared in Fig. 20c, where the related failure loads are reported versus $h_{\mathrm{ef}} / d$ lying between 0 and 35 . Due to the positions of the anchors in the concrete basements, there was no effect of close edges. Based on the indications on the critical edge distances reported in the ETA, splitting failure should be taken into account only for $h_{\mathrm{ef}} d d \geq 33$. For the sake of clarity, the curve for this failure mode was not reported in Fig. 20c.

Pullout failure (Mode P) was predicted based on the following relation (CEN 2018, Eq. 7.13 with coefficients $\psi_{\mathrm{s}, N \mathrm{p}}, \psi_{\mathrm{ec}, N \mathrm{p}}$ and $\psi_{\mathrm{re}, N \mathrm{p}}$ being unitary):

$$
N_{\mathrm{Rm}, \mathrm{p}}=N_{\mathrm{Rm}, \mathrm{p}}^{0} \psi_{\mathrm{g}, N \mathrm{p}}\left(A_{\mathrm{p}, N} / A_{\mathrm{p}, N}^{0}\right)
$$

where $N_{\mathrm{Rm}, \mathrm{p}}^{0}$ coincides with the pullout resistance of one single anchor provided by Eq. (2), coefficient $\psi_{\mathrm{g}, N \mathrm{p}}$ takes account of group effects for closely spaced anchors and ratio 
$A_{\mathrm{p}, N} / A_{\mathrm{p}, N}^{0}$ depends on anchors spacing and edge distances. In particular, coefficient $\psi_{\mathrm{g}, N \mathrm{p}}$ is given by (CEN 2018, Eq. 7.17):

$$
\psi_{\mathrm{g}, N \mathrm{p}}=\psi_{\mathrm{g}, N \mathrm{p}}^{0}-\sqrt{\left(s / s_{\mathrm{cr}, N \mathrm{p}}\right)}\left(\psi_{\mathrm{g}, N \mathrm{p}}^{0}-1\right) \geq 1,
$$

where

$$
\psi_{\mathrm{g}, N \mathrm{p}}^{0}=\sqrt{n}-(\sqrt{n}-1)\left(\tau_{\mathrm{Rm}} / \tau_{\mathrm{Rm}, \max }\right)^{1.5} \geq 1,
$$

$n$ is the number of anchors in the fastening and

$$
\tau_{\mathrm{Rm}, \max }=\left(k_{1} / \pi d\right) \sqrt{h_{\mathrm{ef}} f_{\mathrm{cm}}}
$$

is the upper bound for the bond strength of one single anchor, obtained by equating Eq. (2) with Eq. (3) (Eligehausen et al. 2006a). In Eq. (8) $s$ indicates the mean value of the anchor spacing (FIB 2011), whereas $s_{\mathrm{cr}, N \mathrm{p}}$ takes the form (CEN 2018, Eq. 7.15 with coefficient $\left.\psi_{\text {sus }}=1\right)$ :

$$
s_{\mathrm{cr}, N \mathrm{p}}=7.3 d \sqrt{\tau_{\mathrm{Rk} 20 / 25}} \leq 3 h_{\mathrm{ef}}
$$

with $\tau_{\mathrm{Rk} 20 / 25}$ being the characteristic bond strength for uncracked concrete of class C20/25. In Eq. (7), $A_{\mathrm{p}, N}^{0}=s_{\mathrm{cr}, N \mathrm{p}}^{2}$ is the reference bond influence area on an individual anchor, whereas $A_{\mathrm{p}, N}$ represents the actual bond influence area, limited by overlapping areas of adjacent anchors. A qualitative representation of $A_{\mathrm{p}, N}$ corresponding to the case $s_{\mathrm{cr}, N \mathrm{p}} \geq \min \left\{s_{1}, s_{2}\right\}$, with $s_{1}$ and $s_{2}$ anchor spacings into two orthogonal directions (Fig. 21a), is represented in Fig. 21b. Once again, in estimating the failure load corresponding to Mode $\mathrm{P}$, a uniform bond stress distribution was assumed along the embedment depth also for $h_{\text {ef }} \geq 20 d$.

Concrete cone failure (Mode $\mathrm{C}$ ) was predicted based on the following relation (CEN 2018, Eq. 7.1 with coefficients $\psi_{\mathrm{s}, N}, \psi_{\mathrm{ec}, N}, \psi_{\mathrm{re}, N}$ and $\psi_{M, N}$ being unitary):

(a)

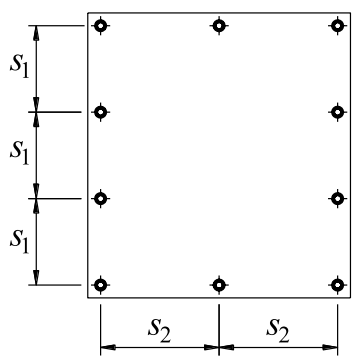

(b)

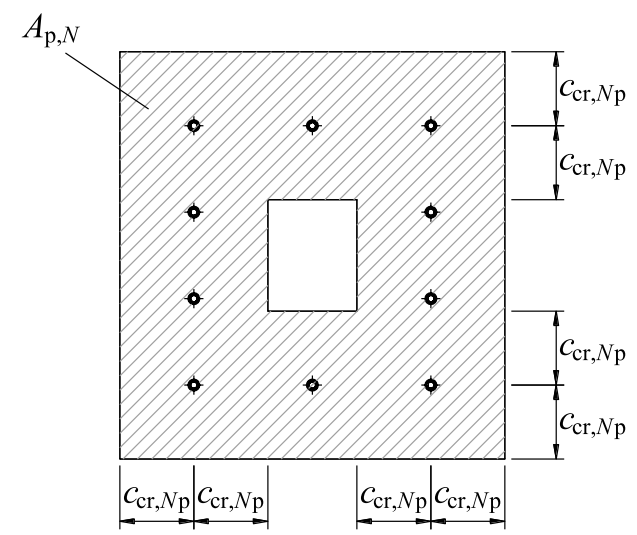

Fig. 21 Group of ten anchors, plan view: $\mathbf{a}$ anchor arrangement; $\mathbf{b}$ effective area $A_{\mathrm{p}, N}$ 


$$
N_{\mathrm{Rm}, \mathrm{c}}=N_{\mathrm{Rm}, \mathrm{c}}^{0}\left(A_{\mathrm{c}, N} / A_{\mathrm{c}, N}^{0}\right)
$$

where $N_{\mathrm{Rm}, \mathrm{c}}^{0}$ coincides with the failure load for concrete cone failure of one single anchor provided by Eq. (3) and ratio $A_{\mathrm{c}, N} / A_{\mathrm{c}, N}^{0}$ takes account of geometric effects due to anchors spacing and edge distances. In Eq. (12), $A_{\mathrm{c}, N}^{0}=s_{\mathrm{cr}, N}^{2}=9 h_{\mathrm{ef}}^{2}$ is the reference projected area for an individual anchor, whereas $A_{\mathrm{c}, N}$ is the actual projected area, limited by overlapping concrete cones of adjacent anchors. Compared with the single anchor case (Fig. 20a), a different trend of concrete breakout failure with respect to pullout failure is observed in Fig. 20c, with the former failure mode appearing as the governing failure mode for a wide range of embedment depths.

Steel failure load (Mode S) was simply obtained by multiplying $N_{\mathrm{Rm}, \mathrm{s}}$ reported in Table 5 by the number of anchors in the fastening. The horizontal line labelled Mode Y in Fig. 20c, differently from the single anchor case, does not refer to yielding of the ten threaded rods, but to yielding of the basement top reinforcement. In fact, mean yielding resistance $N_{\mathrm{ym}}=175 \mathrm{kN}$ of one rod, estimated from Fig. 16 (see Sect. 5.1), would lead to a total load of $1750 \mathrm{kN}$, never achieved in the tests. Therefore, it is then believed that the threaded rods were not yielded in the anchor group tests, and that the changes in slope shown in Fig. 19a, c must rather be ascribed to concrete cracking followed by yielding of the reinforcing bar which were present in the basements. A brief proof of this statement is provided below.

The cracks orthogonal to the beams of the reaction frame (Figs. 17, 18) seem to indicate the onset of a typical flexural failure, such as that usually observed in bending tests. In fact, according to the test configuration shown in Fig. 7b, the basement should be considered as a simply supported beam subjected to concentrated forces in correspondence of the bonded anchors. In the constant moment region between the inner anchors, bending moment is related with total load $N$ by the following equation:

$$
M_{\max }=N_{3} d_{3}+N_{2} d_{2}
$$

where $N_{3}=3 \mathrm{~N} / 10$ and $N_{2}=2 \mathrm{~N} / 10$ indicate the forces due to (three) outer and (two) inner anchors, respectively (see Fig. 13a), whereas $d_{3}=1095 \mathrm{~mm}$ and $d_{2}=1365 \mathrm{~mm}$ are the distances of $N_{3}$ and $N_{2}$ from the closest support. The generic basement cross-section in the constant moment region (Table 6) should then resist $M_{\max }$ given by Eq. (13). Table 7 reports calculated bending moment, concrete strain, neutral axis depth and steel stresses corresponding to total loads reached in tests G1 to G3. In calculations, a parabola-rectangle stress-strain relationship, with strain at the achievement of peak stress $\varepsilon_{\mathrm{c} 2}=0.2 \%$ and ultimate strain $\varepsilon_{\mathrm{cu}}=0.35 \%$ (CEN 2004), was used for concrete in compression, whereas the contribution due to concrete in tension was neglected. For reinforcing steel, an elastic-plastic constitutive law with linear hardening (CEN 2004) was adopted both in tension and in compression, with ultimate strain, yield and ultimate strength coinciding with the mean values reported in Table 2. It is clear from Table 7 that calculations predict yielding of top reinforcing steel $\left(\sigma_{\mathrm{st}} \geq f_{\mathrm{ym}}=495 \mathrm{MPa}\right)$ for all of the tests. It is then to be presumed that, immediately after concrete cracking a stress transfer occurred from concrete to reinforcing bars, which attained yielding in tension for a little greater load. The calculated neutral axis depths appear in line with the measured values, particularly for test G1. The maximum loads reached in the tests, greater than or equal to the failure load corresponding to Mode Y, are also reported in Fig. 20c (diamond data points).

Imposing the achievement of $\varepsilon_{\mathrm{cu}}=0.35 \%$ at the concrete bottom fibre, corresponding to the Ultimate Limit State (ULS) for flexural failure of the basements, the total load 
Table 6 Parameters used for calculations reported in Table 7

\begin{tabular}{|c|c|c|c|}
\hline \multicolumn{2}{|c|}{ Symbol, description (Unit) } & \multirow[t]{2}{*}{ Equation } & \multirow{2}{*}{$\frac{\text { Value }}{3800}$} \\
\hline$B$ & Cross-section breadth (mm) & & \\
\hline$H$ & Cross-section depth (mm) & & 700 \\
\hline$\varnothing_{1}$ & Diameter of principal reinforcement (mm) & & 16 \\
\hline$\varnothing_{2}$ & Diameter of secondary reinforcement $(\mathrm{mm})$ & & 16 \\
\hline$c$ & Clear concrete cover $(\mathrm{mm})$ & & 34 \\
\hline$c_{1 \text { eff }}$ & Effective cover of principal reinforcement $(\mathrm{mm})$ & $c_{1 \mathrm{eff}}=c+\varnothing_{2}+\varnothing_{1} / 2^{\mathrm{a}}$ & 58 \\
\hline$n_{1}$ & Number of principal reinforcing bars within $B(\mathrm{~mm})$ & & 16 \\
\hline$A_{1 \mathrm{~s}}$ & Total area of principal reinforcement $\left(\mathrm{mm}^{2}\right)$ & $A_{1 \mathrm{~s}}=n_{1} \pi \varnothing_{1}^{2} / 4$ & 3217 \\
\hline$f_{\mathrm{cm}}$ & Mean compressive strength of concrete (MPa) & $f_{\mathrm{cm}}=0.83 f_{\mathrm{cm}, \mathrm{cube}}$ & 29.4 \\
\hline
\end{tabular}

${ }^{\text {a }}$ The outer reinforcing layers of the basements were those of secondary reinforcement (orthogonal to the principal reinforcing bars used in calculations reported in Table 7)

Table 7 Calculated maximum bending moment $\left(M_{\max }\right)$ and corresponding concrete strain $\left(\varepsilon_{\mathrm{c}}\right)$, neutral axis depth $(x)$ and stresses in bottom and top reinforcing steel $\left(\sigma_{\mathrm{sb}}, \sigma_{\mathrm{st}}\right)$ for the basements subjected to different load levels $(N)$

\begin{tabular}{lllllll}
\hline Test/limit state & $N$ & $\begin{array}{l}M_{\max } \\
(\mathrm{kNm})\end{array}$ & $\begin{array}{l}\varepsilon_{\mathrm{c}} \\
\left(\times 10^{3}\right)\end{array}$ & $\begin{array}{l}x \\
(\mathrm{~mm})\end{array}$ & $\begin{array}{l}\sigma_{\mathrm{sb}} \\
(\mathrm{MPa})\end{array}$ & $\begin{array}{l}\sigma_{\text {st }} \\
(\mathrm{MPa})\end{array}$ \\
\hline Test G1 & 1627 & 979 & 0.376 & 78 & -19 & 495 \\
Test G2 & 1656 & 996 & 0.547 & 58 & 1 & 497 \\
Test G3 & 1736 & 1044 & 1.182 & 38 & 122 & 508 \\
ULS & 1917 & 1153 & 3.500 & 35 & 451 & 540 \\
\hline
\end{tabular}

results only $10 \%$ greater than that reached in test G3 (last row in Table 7). This failure load, reported in Fig. 20c with a red horizontal line (Mode F), is 10\% smaller than the steel failure load (Mode $\mathrm{S}$ ).

The design failure loads corresponding to the same modes described above (with the exception of Mode Y) are reported in Fig. 20d. Due to the difference in the partial safety factors for materials, the failure load corresponding to Mode $\mathrm{S}$ becomes slightly smaller than that related with Mode F. Disregarding Mode C for the reasons explained in Sect. 6 (the relevant curve is plotted for $h_{\mathrm{ef}} / d \leq 13$ only), the design resistance for the anchor group coincides with steel failure load. It is interesting to note that the design resistance for the fastening results to be unaltered for $h_{\mathrm{ef}} f d \geq 22$. Then, the embedment depth of threaded rods used to anchor the new warehouse to the existing foundation could have been assumed equal to $h_{\mathrm{ef}}=440 \mathrm{~mm}$, corresponding to the intersection between the curves for Mode $\mathrm{S}$ and Mode P in Fig. 20d.

\section{Discussion}

Based on a recent study on column-to-foundation connections, Mahrenholtz and Eligehausen (2016) observed that cyclic loading does not affect the connection capacity of anchorages experiencing yielding of the starter bars. Analogously, in tests on single anchors presented in this study, where threaded rods always experienced yielding, the effects due to the adopted loading protocol were negligible. Conversely, in the absence of 
Fig. 22 Coefficient $\psi_{\mathrm{g}, N \mathrm{p}}^{0}$ versus nondimensional bond strength $\tau_{\mathrm{Rm}} / \tau_{\mathrm{Rm} \text { max }}$

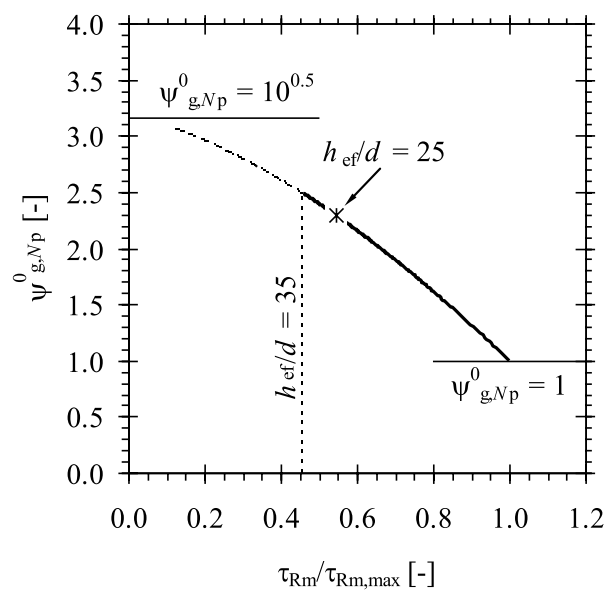

yielding of the threaded rods, failure loads for Mode $\mathrm{P}$ and Mode $\mathrm{C}$ may be affected by cyclic loading (Mahrenholtz 2012; Mahrenholtz et al. 2014; Mahrenholtz and Eligehausen 2016). However, the reduction in the fastening resistance due to factor $\psi_{\text {cyc }, N}$ defined by Mahrenholtz and Eligehausen (2016) for bonded rebars is not greater than 10\%, and for $20 \mathrm{~mm}$-diameter threaded rods would have no effect for $h_{\mathrm{ef}} / d>22$. It was then decided not to apply this reduction factor, in Fig. 20c, to failure loads evaluated for the anchor group.

With regard to Mode P [Eqs. (2) and (7) reported above], the mean bond strength of the adhesive should have been determined by single anchor tests using higher strength steel and an embedment length that would preclude steel failure. The characteristic bond strength reported in the ETA typically is a lower bound number, since it has been adjusted downward to account for performance in reliability and service condition tests. Likely, the actual mean bond strength of the product is around $18 \mathrm{MPa}$ and not the $12.6 \mathrm{MPa}$ assumed (Table 2). This would mean that assuming $h_{\mathrm{ef}}=350 \mathrm{~mm}\left(h_{\mathrm{ef}} / d=17.5\right)$ could well have shown the same test results as the fastening with $h_{\mathrm{ef}}=500 \mathrm{~mm}$. That said, the evaluation of the bond strength of the adhesive was out of the scope of this research, which was aimed at investigating the fastening exactly as it was defined by the design of the new warehouse.

The maximum load reached in the tests on the anchor groups is $13 \%$ greater than predicted failure load for Mode C [Eq. (12)], and no sign of concrete breakout failure was observed. For the fastening investigated, the concrete cone failure load predicted by CEN (2018) appears then too conservative and increasing the applied load would have probably led to the flexural failure of the basements. The failure load according with Mode $\mathrm{C}$ results significantly smaller than that corresponding to Mode $\mathrm{P}$ essentially due to ratio $A_{\mathrm{c}, N} / A_{\mathrm{c}, N}^{0}$, which rapidly decreases for increasing $h_{\mathrm{ef}}$, and is smaller than ratio $A_{\mathrm{p}, N} / A_{\mathrm{p}, N}^{0}$ [right-hand side of Eq. (7)] for $h_{\mathrm{ef}} / d \geq 8$. For example, for $h_{\mathrm{ef}} / d=25, A_{\mathrm{c}, N} / A_{\mathrm{c}, N}^{0}=2.3$, whereas $A_{\mathrm{p}, N} / A_{\mathrm{p}, N}^{0}=6.7$.

For anchor groups with a theoretical spacing $s=0$, coefficient $\psi_{\mathrm{g}, N \mathrm{p}}^{0}$ given by Eq. (9) takes account of the increase of the failure surface when the failure is governed by Mode P. This increase tends to vanish when the failure is controlled by Mode $\mathrm{C}$ (Eligehausen et al. 2006a). Upper and lower bounds for $\psi_{\mathrm{g}, N \mathrm{p}}^{0}$ are then $\sqrt{n}$ for $\tau_{\mathrm{Rm}} / \tau_{\mathrm{Rm}, \max }=0$ (pullout failure) and 1 for $\tau_{\mathrm{Rm}} / \tau_{\mathrm{Rm}, \max }=1$ (concrete cone failure). Coefficient $\psi_{\mathrm{g}, N \mathrm{p}}^{0}$ is plotted in Fig. 22 ver- 
sus ratio $\tau_{\mathrm{Rm}} / \tau_{\mathrm{Rm} \text {,max }}$, with $\tau_{\mathrm{Rm} \text {,max }}$ provided by Eq. (10). It is worth observing that for the embedment depth used in the experiments $\left(h_{\mathrm{ef}} / d=25\right)$ ratio $\tau_{\mathrm{Rm}} / \tau_{\mathrm{Rm}, \max }=0.55$, indicating that a mixed failure mode combining pullout and concrete cone failure, is to be expected. Figure 16 reported by Eligehausen et al. (2006a), concerning groups of $n=4$ anchors, shows that for most of the numerical analyses carried out with $\tau_{\mathrm{Rm}} / \tau_{\mathrm{Rm}, \max } \geq 0.75$ a substantially unit value of $\psi_{\mathrm{g}, N \mathrm{p}}^{0}$ was obtained. Conversely, for smaller values of $\tau_{\mathrm{Rm}} / \tau_{\mathrm{Rm}, \max }$ the group effect associated with $\psi_{\mathrm{g}, N \mathrm{p}}^{0}$ appears significant. With regard to material properties adopted in the present research, a ratio $\tau_{\mathrm{Rm}} / \tau_{\mathrm{Rm} \text { max }} \geq 0.75$ would correspond to $h_{\mathrm{ef}} / d \leq 13$. For larger embedment depths, Mode $\mathrm{C}$ does not appear particularly pertinent. In addition, if spacing $s$ is increased, at equal $h_{\mathrm{ef}}$, up to assume practical values, Mode $\mathrm{C}$ becomes increasingly less probable (Eligehausen et al. 2006a). To highlight this feature, the curve corresponding to Mode $\mathrm{C}$ is represented, in Fig. 20d, only for $h_{\mathrm{ef}} f d \leq 13$ and is reported, in Fig. 20c, in black for $h_{\mathrm{ef}} d d \leq 13$ and in grey for $h_{\mathrm{ef}} / d>13$.

To further support the exclusion of Mode C, $N_{\mathrm{Rm}, \mathrm{c}}$ [see Eq. (12)] may be estimated calculating term $N_{\mathrm{Rm}, \mathrm{c}}^{0}$ according to Eq. (9b) reported by Fuchs et al. (1995). This equation, derived applying the Concrete Capacity Design (CCD) approach to one single anchor in uncracked concrete and far from edges, coincides with Eq. (3) provided that $f_{\mathrm{cm}}$ is replaced with the compression strength measured on cubes with the side length of $200 \mathrm{~mm}$ and $k_{1}=13.5$. With these substitutions into Eq. (3), Eq. (12) provides concrete breakout strengths $35 \%$ larger than those reported in Fig. 20c. In conclusion, Mode C should not be used to interpret the experimental results, which are well approximated by Mode Y due to the large embedment depth adopted.

This feature confirms that the failure of the tested fastening was controlled by the flexural strength of the foundation and also indicates that a reduced embedment depth could have been used.

The load causing cracking of the top surface of the basements in the tests on the anchor groups was of $1498 \mathrm{kN}$ (Fig. 19a, c). The presence of reinforcing steel in the basements not only led to an increase in the fastening resistance (the maximum load achieved in test G3 is $16 \%$ greater than the cracking load), but also in ductility. For example, for test G3 the ratio between the mean vertical displacements of the fastening at maximum and cracking loads results $5.2 / 1.4=3.6$.

The overstrength factor for the anchor groups, calculated as the ratio between the maximum load achieved in the tests and design resistance $N_{\mathrm{Rd}, \mathrm{s}}=1307 \mathrm{kN}$, is equal to 1.33 .

\section{Conclusions}

Unconfined pullout tests on bonded anchors post-installed into initially uncracked, RC foundations are described in the paper. Threaded rods with diameter $d=20 \mathrm{~mm}$ were used as anchors. Materials and products, qualified by the manufacturer for use in seismic zone, belong to seismic performance category C2. The available design equations (FIB 2011; CEN 2018) cover fastenings with up to nine anchors and embedment depth lying in the range $90 \mathrm{~mm}(=4.5 d) \leq h_{\mathrm{ef}} \leq 400 \mathrm{~mm}(=20 d)$.

Eight single-anchor tests were initially conducted, four of which with $h_{\mathrm{ef}}=500 \mathrm{~mm}$ $(=25 d)$ and the remaining four with $h_{\mathrm{ef}}=550 \mathrm{~mm}(=27.5 d)$. For three of the tests with $h_{\mathrm{ef}}=500 \mathrm{~mm}$ and one of those with $h_{\mathrm{ef}}=550 \mathrm{~mm}$, a cyclic loading protocol was used. For all other tests, the tensile force was applied monotonically. For two of the monotonic tests with $h_{\mathrm{ef}}=550 \mathrm{~mm}$, the actuator stroke length was achieved with the rod resisting a force 
about $2 \%$ greater than the expected steel failure resistance. In all other tests, rod failure was attained at very barely scattered loads. Therefore, the difference in the embedment depths adopted in the tests did not play any significant role. Concrete cracking observed at the end of tests on the foundation surface, more pronounced in the case of cyclic loading, consisted in almost circular cracks with a diameter of approximately $160 \mathrm{~mm}$ in the case of 6-cycles loading protocol, and up to $400 \mathrm{~mm}$ for 10-cycles protocol. The mean value of the rod maximum displacement obtained from monotonic tests was $27 \%$ greater than that obtained from cyclic tests.

Three unconfined pullout tests on fastenings comprised of ten bonded anchors with $h_{\mathrm{ef}}=500 \mathrm{~mm}$, analogous to those used for single-anchor tests, were then carried out. Due to the large embedment depth, number of anchors and their positioning, the design of these fastenings was not supported by current European design guide (CEN 2018). The experiments were intended to analyze the response to cyclic tensile loads of the connection of a new automated pallet warehouse with an existing RC foundation slab.

The anchors configuration of the fastening was imposed by the design of the warehouse. The staff of the Laboratory of Structural Engineering of the University of Ferrara was commissioned of the experimental activity necessary to validate that particular design. Therefore, it was decided to adopt, in the group tests, exactly the same configuration as defined by the design. It is worth noting that, being the embedment depth investigated $25 \%$ larger than the recommended upper bound of $20 d$, the anchorage would have been impractical in the absence of experimental tests demonstrating the validity of the design.

In order not to damage the existing foundation, two new RC basements were realized with dimensions $3800 \times 3800 \times 700 \mathrm{~mm}$ and reinforcement ratios in the two principal directions equal to those present in the existing slab. Two of the tests were conducted with a step-wise increasing cyclic load protocol and assuming initially uncracked concrete. In the third one, a single load cycle was performed on a previously damaged basement. In the two cyclic tests, extensive concrete cracking was observed. Most of the observed cracks clearly indicated the onset of flexural failure of the basements. The single-cycle test, even in the presence of cracks first opened in a previous cyclic test, did not highlight any resistance reduction.

Analytical calculations of the bending capacity of the basements showed that, at the maximum loads achieved in the tests, the top reinforcement was likely to be yielded and hardened. Flexural failure of the basements, which was predicted corresponding to a load of $1917 \mathrm{kN}$ ( $10 \%$ greater than the load of $1736 \mathrm{kN}$ reached in test G3), should then be considered as the most probable failure mode. In the absence of reinforcing steel the basements would collapse at the achievement of the cracking load, $1498 \mathrm{kN}$. The overstrength achieved with respect to the cracking load was then equal to $1736 / 1498=1.16$, whereas the maximum potential overstrength is $1917 / 1498=1.28$. Moreover, the presence of reinforcing steel ensured a gain in ductility of $360 \%$.

The fact that the controlling failure mode of the fastening corresponds to the flexural failure of the foundation strictly depends on specific parameters, such as spacing of the piles, distances between anchors and piles, thickness and reinforcement ratio of the foundation slab, which are typical of the case study analyzed. This confirms that the experiments were necessary to highlight the behaviour of the fastening.

After an examination of all possible failure modes predicted by available design equations, it was found that the design resistance for the anchor group corresponds to steel failure of the anchors. With respect to this resistance, the maximum overstrength reached in the tests is 1.33 . The tested fastening seems then more than adequate to resist the design tensile load due to seismic action. 
Acknowledgements The contribution of Mr. Roberto Mazza to the preparation of the experimental tests is gratefully acknowledged.

Funding Open access funding provided by Università degli Studi di Ferrara within the CRUI-CARE Agreement. The described tests were conducted at the factory of Ceramica Sant'Agostino spa in Sant'Agostino, Italy, using bonded anchors produced and installed by Bossong spa, Grassobbio, Italy. The steel members needed for assembling the reaction frame were provided by Stahlbau Pichler srl, Bolzano, Italy. The tests on single anchors were financed by Stahlbau Pichler srl, whereas those on anchor groups were financed by Ceramica Sant'Agostino spa.

Open Access This article is licensed under a Creative Commons Attribution 4.0 International License, which permits use, sharing, adaptation, distribution and reproduction in any medium or format, as long as you give appropriate credit to the original author(s) and the source, provide a link to the Creative Commons licence, and indicate if changes were made. The images or other third party material in this article are included in the article's Creative Commons licence, unless indicated otherwise in a credit line to the material. If material is not included in the article's Creative Commons licence and your intended use is not permitted by statutory regulation or exceeds the permitted use, you will need to obtain permission directly from the copyright holder. To view a copy of this licence, visit http://creativecommons.org/licenses/by/4.0/.

\section{References}

Arslan T (2016) Group effect in axially loaded chemical anchors embedded in low strength concrete. M.Sc. thesis, İzmir Kâtip Çelebi University, Balatçik

Ballarini R, Yueyue X (2017) Fracture mechanics model of anchor group breakout. J Eng Mech 143(4):04016125

Belleri A, Marini A, Riva P, Nascimbene R (2017) Dissipating and re-centring devices for portal-frame precast structures. Eng Struct 150:736-745

Bokor B, Sharma A, Hofmann J (2019a) Experimental investigations on concrete cone failure of rectangular and non-rectangular anchor groups. Eng Struct 188:202-217

Bokor B, Sharma A, Hofmann J (2019b) Spring modelling approach for evaluation and design of tension loaded anchor groups in case of concrete cone failure. Eng Struct 197:109414

Bournas DA, Negro P, Taucer F (2014) Performance of industrial buildings during the Emilia earthquakes in Northern Italy and recommendations for their strengthening. Bull Earthq Eng 12(5):2383-2404

Braga F, Gigliotti R, Monti G, Morelli F, Nuti C, Salvatore W, Vanzi I (2014) Speedup of post earthquake community recovery: the case of precast sindustrial buildings after the Emilia 2012 earthquake. Bull Earthq Eng 12(5):2405-2418

Buratti N, Minghini F, Ongaretto E, Savoia M, Tullini N (2017) Empirical seismic fragility for the precast RC industrial buildings damaged by the 2012 Emilia (Italy) earthquakes. Earthq Eng Struct Dyn 46(14):2317-2335

Caprili S, Morelli F, Salvatore W, Natali A (2018) Design and analysis of automated rack supported warehouses. Open Civ Eng J 12:150-166

Carydis P, Castiglioni CA, Lekkas E, Kostaki I, Lebesis N, Drei A (2012) The Emilia Romagna, May 2012 earthquake sequence. The influence of the vertical earthquake component and related geoscientific and engineering aspects. Ingegneria Sismica-Int J Earthq Eng XXIX(2-3):31-58

Castiglioni CA (2016) Seismic Behaviour of Steel Storage Pallet Racking Systems. Springer, Research for Development, ISBN 978-3-319-28466-8 (eBook)

Castiglioni CA, Kanyilmaz A et al (2014) Seismic Behaviour of Steel Storage Pallet Racking Systems (SEISRACKS2), European Commission, Research Fund for Coal and Steel, Final Report, EUR 27583 EN. https://doi.org/10.2777/931597, ISBN 978-92-79-53896-4, ISSN 1018-5593, KI-NA-27-583-EN-C

Castiglioni CA, Kanyilmaz A et al (2015) The "SEISRACKS2" EU-RFCS research project "Seismic Behaviour of Steel Storage Pallet Racking Systems.” Costruzioni Metalliche 1:37-48

CEN (European Committee for Standardization) (2004) EN 1992-1-1:2004, Eurocode 2-Design of concrete structures-Part 1-1: general rules and rules for buildings. Brussels

CEN (European Committee for Standardization) (2018) EN 1992-4:2018, Eurocode 2-Design of concrete structures-Part 4: design of fastenings for use in concrete. Brussels

Cook RA (1993) Behavior of chemically bonded anchors. J Struct Eng 119(9):2744-2762 
Cook RA, Klingner RE (1992) Ductile multiple-anchor steel-to-concrete connections. J Struct Eng 118(6): $1645-1665$

Cook RA, Eligehausen R, Appl JJ (2007) Overview: behavior of adhesive bonded anchors. Beton- und Stahlbetonbau 102:16-21 (special ed.)

Dal Lago B, Toniolo G, Felicetti R, Lamperti TM (2017) End support connection of precast roof elements by bolted steel angles. Struct Concr 18(5):755-767

Delhomme F, Roure T, Arrieta B, Limam A (2015) Static and cyclic pullout behavior of cast-in-place headed and bonded anchors with large embedment depths in cracked concrete. Nucl Eng Des 287:139-150

Eligehausen R, Cook RA, Appl JJ (2006a) Behavior and design of adhesive bonded anchors. ACI Struct J 103(6):822-831

Eligehausen R, Mallée R, Silva R (2006b) Anchorage in concrete construction. Wilhelm Ernst \& Sohn, Berlin

EOTA (European Organisation for Technical Assessment) (1997) Guideline for European Technical Approval of metal anchors for use in concrete, ETAG 001-Annex C: Design methods for anchorages. Brussels, 1997 (3rd amended August 2010)

EOTA (European Organisation for Technical Assessment) (2007) Design of bonded anchors, Technical Report TR 29. Brussels (amended September 2010)

EOTA (European Organisation for Technical Assessment) (2013a) Design of metal anchors for use in concrete under seismic actions, Technical Report TR 45. Brussels

EOTA (European Organisation for Technical Assessment) (2013b) Guideline for European Technical Approval of metal anchors for use in concrete, ETAG 001-Annex E: Assessment of metal anchors under seismic actions. Brussels

EOTA (European Organisation for Technical Assessment) (2016a) Details of tests for post-installed fasteners in concrete, Technical Report TR 48. Brussels

EOTA (European Organisation for Technical Assessment) (2016b) Post-installed fasteners in concrete under seismic action, Technical Report TR 49. Brussels

EOTA (European Organisation for Technical Assessment) (2018) Systems for post-installed rebar connections with mortar. EAD 330087-00-0601. http://www.eota.eu Accessed 24 May 2019

Epackachi S, Esmaili O, Mirghaderi SR, Behbahani AAT (2015) Behavior of adhesive bonded anchors under tension and shear loads. J Constr Steel Res 114:269-280

Formisano A, Di Lorenzo G, Iannuzzi I, Landolfo R (2017) Seismic vulnerability and fragility of existing Italian industrial steel buildings. Open Civ Eng J 11:1122-1137

Franchi A, Rosati G, Cattaneo S, Crespi PG, Muciaccia G (2009) Experimental investigation on postinstalled metal anchors subjected to seismic loading in R/C members. In: Studies and researchesannual review of structural concrete, pp 277-294. Starrylink, Brescia

Fuchs W, Eligehausen R, Breen JE (1995) Concrete Capacity Design (CCD) approach for fastening to concrete. ACI Struct J 92(1):73-94

Grilli D, Kanvinde A (2015) Tensile strength of embedded anchor groups: tests and strength models. Charles Pankow Foundation-Building Innovation through Research, Draft Final Report, Project 4-11

Guillet T (2011) Behavior of metal anchors under combined tension and shear cycling loads. ACI Struct J 108(3):315-323

Hoehler MS, Eligehausen R (2008a) Behavior and testing of anchors in simulated seismic cracks. ACI Struct J 105(3):348-357

Hoehler MS, Eligehausen R (2008b) Behavior of anchors in cracked concrete under tension cycling at nearultimate loads. ACI Struct J 105(5):601-608

Hoehler MS, Mahrenholtz P, Eligehausen R (2011) Behavior of anchors in concrete at seismic-relevant loading rates. ACI Struct J 108(2):238-247

Hutchinson TC, Wood RL (2013) Cyclic load protocol for anchored nonstructural components and systems. Earthq Spectra 29(3):817-842

Kanyilmaz A, Brambilla G, Chiarelli GP, Castiglioni CA (2016a) Assessment of the seismic behaviour of braced steel storage racking systems by means of full scale push over tests. Thin Walled Struct 107:138-155

Kanyilmaz A, Castiglioni CA, Brambilla G, Chiarelli GP (2016b) Experimental assessment of the seismic behavior of unbraced steel storage pallet racks. Thin-Walled Struct 108:391-405

FIB (International Federation for Structural Concrete) (2011) Design of anchorages in concrete. FIB Bulletin No. 58. Lausanne: FIB

Lehr B (2003) Tragverhalten von Verbunddübeln unter zentrischer Belastung im ungerissenen Beton-Gruppenbefestigungen und Befestigungen am Bauteilrand. Ph.D. thesis, University of Stuttgart 
Liberatore L, Sorrentino L, Liberatore D, Decanini LD (2013) Failure of industrial structures induced by the Emilia (Italy) 2012 earthquakes. Eng Fail Anal 34:629-647

Liu YL, Jiang TZ, Huan JH, Xu FQ (2014) Experimental study on tension performance of anchor group. Adv Mater Res 912-914:666-674

Magliulo G, Ercolino M, Petrone C, Coppola O, Manfredi G (2014) The Emilia earthquake: seismic performance of precast reinforced concrete buildings. Earthq Spectra 30(2):891-912

Magliulo G, Cimmino M, Ercolino M, Manfredi G (2017) Cyclic shear tests on RC precast beam-to-column connections retrofitted with a three-hinged steel device. Bull Earthq Eng 15:3797-3817

Maguire JR, Teh LH, Clifton GC, Tang ZH, Lim JBP (2020) Cross-aisle seismic performance of selective storage racks. J Constr Steel Res 168:105999

Mahrenholtz C (2012) Seismic bond model for concrete reinforcement and the application to column-tofoundation connections. Ph.D. dissertation, University of Stuttgart, Stuttgart

Mahrenholtz C, Akguzel U, Eligehausen R, Pampanin S (2014) New design methodology for seismic column-to-foundation anchorage connections. ACI Struct J 111(5):1179-1189

Mahrenholtz C, Eligehausen R, Reinhardt HW (2015) Design of post-installed reinforcing bars as end anchorage or as bonded anchor. Eng Struct 100:645-655

Mahrenholtz C, Eligehausen R (2016) Simulation of tests on cast-in and postinstalled column-to-foundation connections to quantify the effect of cyclic loading. J Struct Eng 142(1):04015073

Mahrenholtz P, Eligehausen R, Hutchinson TC, Hoehler MS (2016) Behavior of post-installed anchors tested by stepwise increasing cyclic load protocols. ACI Struct J 113(5):997-1008

Mahrenholtz P, Eligehausen R, Hutchinson TC, Hoehler MS (2017a) Behavior of post-installed anchors tested by stepwise increasing cyclic crack protocols. ACI Struct J 114(3):621-630

Mahrenholtz P, Wood RL, Eligehausen R, Hutchinson TC, Hoehler MS (2017b) Development and validation of European guidelines for seismic qualification of post-installed anchors. Eng Struct 148:497-508

Mallée R, Fuchs W, Eligehausen R (2013) Design of fastenings for use in concrete - the CEN/TS 1992/4 provisions. Wilhelm Ernst \& Sohn, Berlin

Minghini F, Ongaretto E, Ligabue V, Savoia M, Tullini N (2016) Observational failure analysis of precast buildings after the 2012 Emilia earthquakes. Earthq Struct 11(2):327-346

Minghini F, Tullini N (2021) Seismic retrofitting solutions for precast RC industrial buildings struck by the 2012 earthquakes in Northern Italy. Front Built Environ 7:631315. https://doi.org/10.3389/fbuil.2021. 631315

Nilforoush R, Nilsson M, Elfgren L, Ožbolt J, Hofmann J, Eligehausen R (2017) Influence of surface reinforcement, member thickness and cracked concrete on tensile capacity of anchor bolts. ACI Struct J 114(6):1543-1556

Nilforoush R, Nilsson M, Elfgren L (2018) Experimental evaluation of influence of member thickness, anchor-head size, and orthogonal surface reinforcement on the tensile capacity of headed anchors in uncracked concrete. J Struct Eng 144(4):04018012

Nuti C, Santini S (2008) Fastening technique in seismic areas: a critical review. In: Walraven JC, Stoelhorst D (eds) Proceedings of the international FIB symposium 2008-Tailor made concrete structures-new solutions for our society (pp. 899-905). CRC Press, Amsterdam

Ozturk M (2013) Prediction of tensile capacity of adhesive anchors including edge and group effects using neural networks. Sci Eng Compos Mater 20(1):95-104

Parastesh H, Hajirasouliha I, Ramezani R (2014) A new ductile moment-resisting connection for precast concrete frames in seismic regions: an experimental investigation. Eng Struct 70:144-157

Pollini AV, Buratti N, Mazzotti C (2018) Experimental and numerical behaviour of dissipative devices based on carbon-wrapped steel tubes for the retrofitting of existing precast RC structures. Earthq Eng Struct Dyn 47:1270-1290

IMIT (Italian Ministry of Infrastructure and Transport) (2018) Italian Building Code-D.M. 17/01/2018. Rome, Italy, 2018 [in Italian]

Rosin I, Castiglioni CA et al (2009) Storage Racks in Seismic Areas (SEISRACKS), European Commission, Research Fund for Coal and Steel, Final Report, EUR 23744 EN. https://doi.org/10.2777/60886, ISBN 978-92-79-11203-4, ISSN 1018-5593, KI-NA-23744-EN-S

Tullini N, Minghini F (2016) Grouted sleeve connections used in precast reinforced concrete constructionexperimental investigation of a column-to-column joint. Eng Struct 127:784-803

Tullini N, Minghini F (2020) Cyclic test on a precast reinforced concrete column-to-foundation grouted duct connection. Bull Earthq Eng 18(4):1657-1691

Węglorz M (2017) Influence of headed anchor group layout on concrete failure in tension. Procedia Engineering 193:242-249

Wood RL, Hutchinson TC (2013) Crack protocols for anchored components and systems. ACI Struct J 110(3):391-402 
Publisher's Note Springer Nature remains neutral with regard to jurisdictional claims in published maps and institutional affiliations. 